TRANSACTIONS OF THE

AMERICAN MATHEMATICAL SOCIETY

Volume 361, Number 11, November 2009, Pages 6073-6114

S 0002-9947(09)04898-3

Article electronically published on June 15, 2009

\title{
SIMPLICIAL MATRIX-TREE THEOREMS
}

\author{
ART M. DUVAL, CAROLINE J. KLIVANS, AND JEREMY L. MARTIN
}

\begin{abstract}
We generalize the definition and enumeration of spanning trees from the setting of graphs to that of arbitrary-dimensional simplicial complexes $\Delta$, extending an idea due to G. Kalai. We prove a simplicial version of the Matrix-Tree Theorem that counts simplicial spanning trees, weighted by the squares of the orders of their top-dimensional integral homology groups, in terms of the Laplacian matrix of $\Delta$. As in the graphic case, one can obtain a more finely weighted generating function for simplicial spanning trees by assigning an indeterminate to each vertex of $\Delta$ and replacing the entries of the Laplacian with Laurent monomials. When $\Delta$ is a shifted complex, we give a combinatorial interpretation of the eigenvalues of its weighted Laplacian and prove that they determine its set of faces uniquely, generalizing known results about threshold graphs and unweighted Laplacian eigenvalues of shifted complexes.
\end{abstract}

\section{INTRODUCTION}

This article is about generalizing the Matrix-Tree Theorem from graphs to simplicial complexes.

1.1. The classical Matrix-Tree Theorem. We begin by reviewing the classical case; for a more detailed treatment, see, e.g., [8]. Let $G$ be a finite, simple, undirected graph with vertices $V(G)=[n]=\{1,2, \ldots, n\}$ and edges $E(G)$. A spanning subgraph of $G$ is a graph $T$ with $V(T)=V(G)$ and $E(T) \subseteq E(G)$; thus a spanning subgraph may be specified by its edge set. A spanning subgraph $T$ is a spanning tree if (a) $T$ is acyclic; (b) $T$ is connected; and (c) $|E(T)|=|V(T)|-1$. It is a fundamental property of spanning trees (the "two-out-of-three theorem") that any two of these three conditions together imply the third.

The Laplacian of $G$ is the $n \times n$ symmetric matrix $L=L(G)$ with entries

$$
L_{i j}= \begin{cases}\operatorname{deg}_{G}(i) & \text { if } i=j, \\ -1 & \text { if } i, j \text { are adjacent } \\ 0 & \text { otherwise, }\end{cases}
$$

where $\operatorname{deg}_{G}(i)$ is the degree of vertex $i$ (the number of edges having $i$ as an endpoint). Equivalently, $L=\partial \partial^{*}$, where $\partial$ is the (signed) vertex-edge incidence matrix

Received by the editors February 27, 2008.

2000 Mathematics Subject Classification. Primary 05A15; Secondary 05E99, 05C05, 05C50, 15A18, 57M15.

Key words and phrases. Simplicial complex, spanning tree, tree enumeration, Laplacian, spectra, eigenvalues, shifted complex.

The second author was partially supported by NSF VIGRE grant DMS-0502215.

The third author was partially supported by an NSA Young Investigators Grant.

(C)2009 American Mathematical Society Reverts to public domain 28 years from publication 
and $\partial^{*}$ is its transpose. If we regard $G$ as a one-dimensional simplicial complex, then $\partial$ is just the simplicial boundary map from 1 -faces to 0 -faces, and $\partial^{*}$ is the simplicial coboundary map. The matrix $L$ is symmetric, hence diagonalizable, so it has $n$ real eigenvalues (counting multiplicities). The number of nonzero eigenvalues of $L$ is $n-c$, where $c$ is the number of components of $G$.

The Matrix-Tree Theorem, first observed by Kirchhoff [22] in his work on electrical circuits (modern references include 8, [29] and 34, Chapter 5]), expresses the number $\tau(G)$ of spanning trees of $G$ in terms of $L$. The theorem has two equivalent formulations.

Theorem 1.1 (Classical Matrix-Tree Theorem). Let $G$ be a connected graph with $n$ vertices, and let $L$ be its Laplacian matrix.

(1) If the eigenvalues of $L$ are $\lambda_{0}=0, \lambda_{1}, \ldots, \lambda_{n-1}$, then

$$
\tau(G)=\frac{\lambda_{1} \cdots \lambda_{n-1}}{n} .
$$

(2) For $1 \leq i \leq n$, let $L_{i}$ be the reduced Laplacian obtained from $L$ by deleting the $i^{\text {th }}$ row and $i^{\text {th }}$ column. Then

$$
\tau(G)=\operatorname{det} L_{i}
$$

Well-known corollaries of the Matrix-Tree Theorem include Cayley's formula [9]

$$
\tau\left(K_{n}\right)=n^{n-2}
$$

where $K_{n}$ is the complete graph on $n$ vertices, and Fiedler and Sedláček's formula 16]

$$
\tau\left(K_{n, m}\right)=n^{m-1} m^{n-1},
$$

where $K_{n, m}$ is the complete bipartite graph on vertex sets of sizes $n$ and $m$.

The Matrix-Tree Theorem can be refined by introducing an indeterminate $e_{i j}=$ $e_{j i}$ for each pair of vertices $i, j$, setting $e_{i j}=0$ if $i, j$ do not share a common edge. The weighted Laplacian $\hat{L}$ is then defined as the $n \times n$ matrix with entries

$$
\hat{L}_{i j}= \begin{cases}\sum_{k=1}^{n} e_{i k} & \text { if } i=j \\ -e_{i j} & \text { if } i, j \text { are adjacent } \\ 0 & \text { otherwise. }\end{cases}
$$

Theorem 1.2 (Weighted Matrix-Tree Theorem). Let $G$ be a graph with $n$ vertices, and let $\hat{L}$ be its weighted Laplacian matrix.

(1) If the eigenvalues of $\hat{L}$ are $\hat{\lambda}_{0}=0, \hat{\lambda}_{1}, \ldots, \hat{\lambda}_{n-1}$, then

$$
\sum_{T \in \mathcal{T}(G)} \prod_{i j \in T} e_{i j}=\frac{\hat{\lambda}_{1} \cdots \hat{\lambda}_{n-1}}{n}
$$

where $\mathcal{T}(G)$ is the set of all spanning trees of $G$.

(2) For $1 \leq i \leq n$, let $\hat{L}_{i}$ be the reduced weighted Laplacian obtained from $\hat{L}$ by deleting the $i^{\text {th }}$ row and $i^{\text {th }}$ column. Then

$$
\sum_{T \in \mathcal{T}(G)} \prod_{i j \in T} e_{i j}=\operatorname{det} \hat{L}_{i} .
$$


By making appropriate substitutions for the indeterminates $e_{i j}$, it is often possible to obtain finer enumerative information than merely the number of spanning trees. For instance, when $G=K_{n}$, introducing indeterminates $x_{1}, \ldots, x_{n}$ and setting $e_{i j}=x_{i} x_{j}$ for all $i, j$ yields the Cayley-Prüfer Theorem, which enumerates spanning trees of $K_{n}$ by their degree sequences:

$$
\sum_{T \in \mathcal{T}(G)} \prod_{i=1}^{n} x_{i}^{\operatorname{deg}_{T}(i)}=x_{1} \cdots x_{n}\left(x_{1}+\cdots+x_{n}\right)^{n-2} .
$$

Note that Cayley's formula (11) can be recovered from the Cayley-Prüfer Theorem by setting $x_{1}=\cdots=x_{n}=1$.

1.2. Simplicial spanning trees and how to count them. To extend the scope of the Matrix-Tree Theorem from graphs to simplicial complexes, we must first say what "spanning tree" means in arbitrary dimension. Kalai [20] proposed a definition that replaces the acyclicity, connectedness, and edge-count conditions with their analogues in simplicial homology. Our definition adapts Kalai's definition to a more general class of simplicial complexes 1

Let $\Delta$ be a $d$-dimensional simplicial complex, and let $\Upsilon \subset \Delta$ be a subcomplex containing all faces of $\Delta$ of dimension $<d$. We say that $\Upsilon$ is a simplicial spanning tree of $\Delta$ if the following three conditions hold:

$$
\begin{aligned}
& \tilde{H}_{d}(\Upsilon, \mathbb{Z})=0, \\
& \left|\tilde{H}_{d-1}(\Upsilon, \mathbb{Z})\right|<\infty, \text { and } \\
& f_{d}(\Upsilon)=f_{d}(\Delta)-\tilde{\beta}_{d}(\Delta)+\tilde{\beta}_{d-1}(\Delta),
\end{aligned}
$$

where $\tilde{H}_{i}$ denotes reduced simplicial homology (for which see, e.g., [18, §2.1]). (The conditions (4a) and (4b) were introduced by Kalai in 20, while (4c) is more general, as we will explain shortly.) When $d=1$, the conditions (4a)... (4c) say respectively that $\Upsilon$ is acyclic, connected, and has one fewer edge than it has vertices, recovering the definition of the spanning tree of a graph. Moreover, as we will show in Proposition 3.5, any two of the three conditions together imply the third.

A graph $G$ has a spanning tree if and only if $G$ is connected. The corresponding condition for a simplicial complex $\Delta$ of dimension $d$ is that $\tilde{H}_{i}(\Delta, \mathbb{Q})=0$ for all $i<d$; that is, $\Delta$ has the rational homology type of a wedge of $d$-dimensional spheres. We will call such a complex acyclic in positive codimension, or APC for short. This condition, which we will assume throughout the rest of the introduction, is much weaker than Cohen-Macaulayness (by Reisner's theorem 30]), and therefore encompasses many complexes of combinatorial interest, including all connected graphs, simplicial spheres, shifted, matroid, and Ferrers complexes, and some chessboard and matching complexes.

For $k \leq d$, let $\partial=\partial_{k}$ be the $k$ th simplicial boundary matrix of $\Delta$ (with rows and columns indexed respectively by $(k-1)$-dimensional and $k$-dimensional faces of $\Delta)$, and let $\partial^{*}$ be its transpose. The (kth up-down) Laplacian of $\Delta$ is $L=\partial \partial^{*}$; this can

\footnotetext{
${ }^{1}$ There are many other definitions of "simplicial tree" in the literature, depending on which properties of trees one wishes to extend; see, e.g., 4, 10, 15, 19, 28. By adopting Kalai's idea, we choose a definition that lends itself well to enumeration. The closest to ours in spirit is perhaps that of Masbaum and Vaintrob [28, whose main result is a Matrix-Tree-like theorem enumerating a different kind of 2-dimensional tree using Pfaffians rather than Laplacians.
} 
be regarded either as a square matrix of size $f_{k-1}(\Delta)$ or as a linear endomorphism on $(k-1)$-chains of $\Delta$. Define invariants

$$
\begin{aligned}
\pi_{k}=\pi_{k}(\Delta) & =\text { product of all nonzero eigenvalues of } L, \\
\tau_{k}=\tau_{k}(\Delta) & =\sum_{\Upsilon \in \mathcal{T}_{k}(\Delta)}\left|\tilde{H}_{k-1}(\Upsilon)\right|^{2},
\end{aligned}
$$

where $\mathcal{T}_{k}(\Delta)$ denotes the set of all $k$-trees of $\Delta$ (that is, simplicial spanning trees of the $k$-skeleton of $\Delta$ ).

Kalai [20] studied these invariants in the case that $\Delta$ is a simplex on $n$ vertices, and proved the formula

$$
\tau_{k}(\Delta)=n^{\left(\begin{array}{c}
n-2 \\
k
\end{array}\right)}
$$

(of which Cayley's formula (11) is the special case $k=1$ ). Kalai also proved a natural weighted analogue of (5) enumerating simplicial spanning trees by their degree sequences, thus generalizing the Cayley-Prüfer Theorem (3).

Given disjoint vertex sets $V_{1}, \ldots, V_{r}$ ("color classes"), the faces of the corresponding complete colorful complex $\Gamma$ are those sets of vertices with no more than one vertex of each color. Equivalently, $\Gamma$ is the simplicial join $V_{1} * V_{2} * \cdots * V_{r}$ of the 0 -dimensional complexes $V_{i}$. Adin [1] extended Kalai's work by proving a combinatorial formula for $\tau_{k}(\Gamma)$, which we shall not reproduce here, for every $1 \leq k<r$. Note that when $r=2$, the complex $\Gamma$ is a complete bipartite graph, and if $\left|V_{i}\right|=1$ for all $i$, then $\Gamma$ is a simplex. Thus both (2) and (5) can be recovered from Adin's formula.

Kalai's and Adin's beautiful formulas inspired us to look for more results concerning simplicial spanning tree enumeration, and in particular to formulate a simplicial version of the Matrix-Tree Theorem that could be applied to as broad a class of complexes as possible. Our first main result generalizes the Matrix-Tree Theorem to all APC simplicial complexes.

Theorem 1.3 (Simplicial Matrix-Tree Theorem). Let $\Delta$ be a d-dimensional APC simplicial complex. Then:

(1) We have

$$
\pi_{d}(\Delta)=\frac{\tau_{d}(\Delta) \tau_{d-1}(\Delta)}{\left|\tilde{H}_{d-2}(\Delta)\right|^{2}}
$$

(2) Let $U$ be the set of facets of a $(d-1)$-SST (simplicial spanning tree) of $\Delta$, and let $L_{U}$ be the reduced Laplacian obtained by deleting the rows and columns of $L$ corresponding to $U$. Then

$$
\tau_{d}(\Delta)=\frac{\left|\tilde{H}_{d-2}(\Delta)\right|^{2}}{\left|\tilde{H}_{d-2}\left(\Delta_{U}\right)\right|^{2}} \operatorname{det} L_{U}
$$

We will prove these formulas in Section 4

In the special case $d=1$, the number $\tau_{1}(\Delta)$ is just the number of spanning trees of the graph $\Delta$, recovering the classical Matrix-Tree Theorem. When $d \geq 2$, there can exist spanning trees with finite but nontrivial homology groups (the simplest example is the real projective plane). In this case, $\tau_{k}(\Delta)$ is greater than the number of spanning trees, because these "torsion trees" contribute more than 1 to the count. This phenomenon was first observed by Bolker [7, and arises also in the study of cyclotomic matroids [26] and cyclotomic polytopes [3]. 
The Weighted Matrix-Tree Theorem also has a simplicial analogue. Introduce an indeterminate $x_{F}$ for each facet (maximal face) $F \in \Delta$, and for every set $T$ of facets define monomials $x_{T}=\prod_{F \in T} x_{F}$ and $X_{T}=x_{T}^{2}$. Construct the weighted boundary matrix $\hat{\partial}$ by multiplying each column of $\partial$ by $x_{F}$, where $F$ is the facet of $\Delta$ corresponding to that column. Let $\hat{\pi}_{k}$ be the product of the nonzero eigenvalues of $\hat{L}_{\Delta, k-1}^{\mathrm{ud}}$, and let

$$
\hat{\tau}_{k}=\hat{\tau}_{k}(\Delta)=\sum_{\Upsilon \in \mathcal{T}_{k}(\Delta)}\left|\tilde{H}_{k-1}(\Upsilon)\right|^{2} X_{\Upsilon}
$$

Theorem 1.4 (Weighted Simplicial Matrix-Tree Theorem). Let $\Delta$ be a d-dimensional APC simplicial complex. Then:

(1) We have2

$$
\hat{\pi}_{d}(\Delta)=\frac{\hat{\tau}_{d}(\Delta) \tau_{d-1}(\Delta)}{\left|\tilde{H}_{d-2}(\Delta)\right|^{2}}
$$

(2) Let $U$ be the set of facets of a $(d-1)$-SST of $\Delta$, and let $\hat{L}_{U}$ be the reduced Laplacian obtained by deleting the rows and columns of $\hat{L}$ corresponding to U. Then

$$
\hat{\tau}_{d}(\Delta)=\frac{\left|\tilde{H}_{d-2}(\Delta)\right|^{2}}{\left|\tilde{H}_{d-2}\left(\Delta_{U}\right)\right|^{2}} \operatorname{det} \hat{L}_{U}
$$

We will prove these formulas in Section 5 .

Setting $x_{F}=1$ for all $F$ in Theorem 1.4 recovers Theorem 1.3. In fact, more is true; setting $x_{F}=1$ in the multiset of eigenvalues of the weighted Laplacian (reduced or unreduced) yields the eigenvalues of the corresponding unweighted Laplacian. If the complex $\Delta$ is Laplacian integral, that is, its Laplacian matrix has integer eigenvalues, then we can hope to find a combinatorial interpretation of the factorization of $\hat{\tau}_{d}(\Delta)$ furnished by Theorem [1.4. An important class of Laplacian integral simplicial complexes is the class of shifted complexes.

1.3. Results on shifted complexes. Let $p \leq q$ be integers, and let $[p, q]=\{i \in$ $\mathbb{Z}: p \leq i \leq q\}$. A simplicial complex $\Sigma$ on a vertex set $[p, q]$ is shifted if the following condition holds: whenever $i<j$ are vertices and $F \in \Sigma$ is a face such that $i \notin F$ and $j \in F$, then $F \backslash\{j\} \cup\{i\} \in \Sigma$. Equivalently, define the componentwise partial order $\preceq$ on finite sets of positive integers as follows: $A \preceq B$ whenever $A=\left\{a_{1}<\cdots<a_{m}\right\}$, $B=\left\{b_{1}<\cdots<b_{m}\right\}$, and $a_{i} \leq b_{i}$ for all $i$. Then a complex is shifted precisely when it is an order ideal with respect to the componentwise partial order. (See [33, chapter 3] for general background on partially ordered sets.)

Shifted complexes were used by Björner and Kalai [5 to characterize the $f$ vectors and Betti numbers of all simplicial complexes. Shifted complexes are also one of a small handful of classes of simplicial complexes whose Laplacian eigenvalues are known to be integral. In particular, Duval and Reiner [13, Thm. 1.1] proved that the Laplacian eigenvalues of a shifted complex $\Sigma$ on $[p, q]$ are given by the conjugate of the partition $\left(d_{p}, d_{p+1}, \ldots, d_{q}\right)$, where $d_{i}$ is the degree of vertex $i$, that is, the number of facets containing it.

\footnotetext{
${ }^{2}$ Despite appearances, there are no missing hats on the right-hand side of this formula! Only $\tau_{d}(\Delta)$ has been replaced with its weighted analogue; $\tau_{d-1}(\Delta)$ is still just an integer.
} 
In the second part of the article, Sections 6 10 we study factorizations of the weighted spanning tree enumerator of $\Sigma$ under the combinatorial fine weighting

$$
x_{F}=\prod_{i=1}^{k+1} x_{i, v_{i}}
$$

(described in more detail in Section [6), where $F=\left\{v_{1}<\cdots<v_{k+1}\right\}$ is a $k$ dimensional face of $\Sigma$. Thus the term of $\tau_{k}(\Sigma)$ corresponding to a particular simplicial spanning tree of $\Sigma$ contains more precise information than its vertex degrees alone (which can be recovered by further setting $x_{i, j}=x_{j}$ for all $i, j$ ).

For integer sets $A$ and $B$ as above, we call the ordered pair $(A, B)$ a critical pair of $\Sigma$ if $A \in \Sigma, B \notin \Sigma$, and $B$ covers $A$ in the componentwise order. That is, $B=\left\{a_{1}, \ldots, a_{i-1}, a_{i}+1, a_{i+1}, \ldots, a_{m}\right\}$ for some $i \in[m]$. The long signature of $(A, B)$ is the ordered pair $\bar{\sigma}(A, B)=(S, T)$, where $S=\left\{a_{1}, \ldots, a_{i-1}\right\}$ and $T=\left[p, a_{i}\right]$. The corresponding $z$-polynomial is defined as

$$
z(S, T)=\frac{1}{\uparrow X_{S}} \sum_{j \in T} X_{S \cup j}
$$

where $X_{S}=x_{S}^{2}$ for each $S$, and the operator $\uparrow$ is defined by $\uparrow\left(x_{i, j}\right)=x_{i+1, j}$. (See Section 8.1 for more details, and Example 1.7 for an example.) The set of critical pairs is especially significant for a shifted family (and by extension, for a shifted complex). Since a shifted family is just an order ideal with respect to the componentwise partial order $\preceq$, the critical pairs identify the frontier between members and nonmembers of $\mathcal{F}$ in the Hasse diagram of $\preceq$. (See Example 1.7 or 23] for more details.)

Thanks to Theorem 1.4 the enumeration of SST's of a shifted complex reduces to computing the determinant of the reduced combinatorial finely-weighted Laplacian. We show in Section 6 how this computation reduces to the computation of the eigenvalues of the algebraic finely weighted Laplacian. This modification of the combinatorial fine weighting, designed to endow the chain groups of $\Sigma$ with the structure of an algebraic chain complex, is described in detail in Section 6.3. Its eigenvalues turn out to be precisely the $z$-polynomials associated with critical pairs.

Theorem 1.5. Let $\Sigma$ be a d-dimensional shifted complex, and let $0 \leq i \leq d$. Then the eigenvalues of the algebraic finely weighted up-down Laplacian $\mathbf{L}_{\Delta, i}^{\mathrm{ud}}$ are precisely $\uparrow^{d-i}(z(S, T))$, where $(S, T)$ ranges over all long signatures of critical pairs of $i$-dimensional faces of $\Sigma$.

In turn, the $z$-polynomials are the factors of the weighted simplicial spanning tree enumerator $\hat{\tau}_{d}$.

Theorem 1.6. Let $\Sigma$ be a d-dimensional shifted complex with initial vertex $p$. Then:

$$
\begin{aligned}
\hat{\tau}_{d}(\Sigma) & =\left(\prod_{F \in \Lambda_{d-1}} X_{\tilde{F}}\right)\left(\prod_{(S, T) \in \bar{\sigma}\left(\Delta_{d}\right)} \frac{z(S, \tilde{T})}{X_{1, p}}\right) \\
& =\left(\prod_{F \in \Lambda_{d-1}} X_{\tilde{F}}\right)\left(\prod_{(S, T) \in \bar{\sigma}\left(\Delta_{d}\right)} \frac{\sum_{j \in \tilde{T}} X_{S \cup j}}{X_{\tilde{S}}}\right)
\end{aligned}
$$



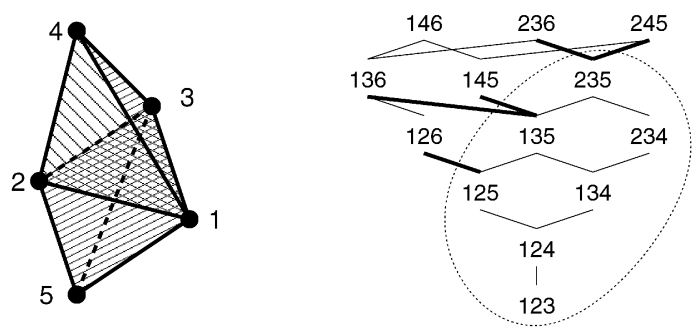

Figure 1

where $\tilde{F}=F \cup\{p\} ; \Delta=\operatorname{del}_{p} \Sigma=\{F \backslash\{p\}: F \in \Sigma\} ;$ and $\Lambda=\operatorname{link}_{p} \Sigma=\{F: p \notin$ $F, \tilde{F} \in \Sigma\}$.

Theorems 1.5 and 1.6 are proved in Sections 8 and 9, respectively.

Example 1.7. As an example to which we will return repeatedly, consider the equatorial bipyramid, the two-dimensional shifted complex $B$ with vertices [5] and facets $123,124,125,134,135,234,235$. A geometric realization of $B$ is shown in Figure 1 on the left. Figure 1 on the right illustrates how the facets of $B$ can be regarded as an order ideal. The boldface lines indicate critical pairs. The Laplacian eigenvalues corresponding to the critical pairs of $B$ are as follows:

\begin{tabular}{|c||c|c|c|c|c|}
\hline Critical pair & $(125,126)$ & $(135,136)$ & $(135,145)$ & $(235,236)$ & $(235,245)$ \\
\hline Eigenvalue & $z(12,12345)$ & $z(13,12345)$ & $z(1,123)$ & $z(23,12345)$ & $z(2,123)$ \\
\hline
\end{tabular}

To show one of these eigenvalues in more detail, we have $z(13,12345)=$

$$
\frac{X_{1,1} X_{2,1} X_{3,3}+X_{1,1} X_{2,2} X_{3,3}+X_{1,1} X_{2,3} X_{3,3}+X_{1,1} X_{2,3} X_{3,4}+X_{1,1} X_{2,3} X_{3,5}}{X_{2,1} X_{3,3}} .
$$

The eigenvalues of this complex are explained in more detail in Section 8.4. Its spanning trees are enumerated in Examples 9.1 (fine weighting) and 9.3 (coarse weighting).

We prove Theorem 1.5 by exploiting the recursive structure of shifted complexes. As in [13, we begin by calculating the algebraic finely weighted eigenvalues of a near-cone in terms of the eigenvalues of its link and deletion with respect to its apex (Proposition 7.6). We can then write down a recursive formula (Theorem 8.2) for the nonzero eigenvalues of shifted complexes, thanks to their characterization as iterated near-cones, simultaneously showing that these eigenvalues must be of the form $z(S, T)$. Finally, we independently establish a recurrence (Corollary 8.8) for the long signatures of critical pairs of a shifted complex, which coincides with the recurrence for the $z(S, T)$, thus yielding a bijection between nonzero eigenvalues and critical pairs.

Corollary 8.10 shows what the eigenvalues look like in coarse weighting. Passing from weighted to unweighted eigenvalues then easily recovers the Duval-Reiner 
formula for Laplacian eigenvalues of shifted complexes in terms of degree sequences [13, Thm. 1.1]. Similarly, Corollary 9.2 gives the enumeration of SST's of a shifted complex in the coarse weighting.

We are also able to show that the finely-weighted eigenvalues (though not the coarsely-weighted eigenvalues) are enough to recover the shifted complex (Corollary (8.9), or, in other words, that one can "hear the shape" of a shifted complex.

Several known results can be obtained as consequences of the general formula of Theorem 1.6.

- The complete $d$-skeleton of a simplex is easily seen to be shifted, and applying Theorem 1.6 to such complexes recovers Kalai's generalization of the Cayley-Prüfer Theorem.

- The one-dimensional shifted complexes are precisely the threshold graphs, an important class of graphs with many equivalent descriptions (see, e.g., [25]). When $d=1$, Theorem 1.6 specializes to the weighted spanning tree enumerator for threshold graphs proved by Martin and Reiner [26, Thm. 4] and following from an independent result of Remmel and Williamson 31, Thm. 2.4].

- Thanks to an idea of Richard Ehrenborg, the formula for threshold graphs can be used to recover a theorem of Ehrenborg and van Willigenburg [14, enumerating spanning trees in certain bipartite graphs called Ferrers graphs (which are not in general Laplacian integral).

We discuss these corollaries in Section 10.

Some classes of complexes that we think deserve further study include matroid complexes, matching complexes, chessboard complexes and color-shifted complexes. The first three kinds of complexes are known to be Laplacian integral, by theorems of Kook, Reiner and Stanton 24], Dong and Wachs [11, and Friedman and Hanlon [17. respectively. Every matroid complex is Cohen-Macaulay [32, §III.3], hence APC, while matching complexes and chessboard complexes are APC for certain values of their defining parameters (see [6]). Color-shifted complexes, which are a common generalization of Ferrers graphs and complete colorful complexes, are not in general Laplacian integral; nevertheless, their weighted simplicial spanning tree enumerators seem to have nice factorizations.

It is our pleasure to thank Richard Ehrenborg, Vic Reiner, and Michelle Wachs for many valuable discussions. We also thank Andrew Crites and an anonymous referee for their careful reading of the manuscript.

\section{Notation AND DEFinitions}

2.1. Simplicial complexes. Let $V$ be a finite set. A simplicial complex on $V$ is a family $\Delta$ of subsets of $V$ such that

(1) $\emptyset \in \Delta$;

(2) if $F \in \Delta$ and $G \subseteq F$, then $G \in \Delta$.

The elements of $V$ are called vertices of $\Delta$, and the faces that are maximal under inclusion are called facets. Thus a simplicial complex is determined by its set of facets. The dimension of a face $F$ is $\operatorname{dim} F=|F|-1$, and the dimension of $\Delta$ is the maximum dimension of a face (or facet). The abbreviation $\Delta^{d}$ indicates that $\operatorname{dim} \Delta=d$. We say that $\Delta$ is pure if all facets have the same dimension; in this case, a ridge is a face of codimension 1 , that is, dimension $\operatorname{dim} \Delta-1$. 
We write $\Delta_{i}$ for the set of $i$-dimensional faces of $\Delta$, and set $f_{i}(\Delta)=\left|\Delta_{i}\right|$. The $i$-skeleton of $\Delta$ is the subcomplex of all faces of dimension $\leq i$,

$$
\Delta_{(i)}=\bigcup_{-1 \leq j \leq i} \Delta_{j}
$$

and the pure $i$-skeleton of $\Delta$ is the subcomplex generated by the $i$-dimensional faces, that is,

$$
\Delta_{[i]}=\left\{F \in \Delta: F \subseteq G \text { for some } G \in \Delta_{i}\right\} .
$$

We assume that the reader is familiar with simplicial homology; see, e.g., [18, $\S 2.1$. Let $\Delta^{d}$ be a simplicial complex and $-1 \leq i \leq d$. Let $R$ be a ring (if unspecified, assumed to be $\mathbb{Z}$ ), and let $C_{i}(\Delta)$ be the $i^{t h}$ simplicial chain group of $\Delta$, i.e., the free $R$-module with basis $\left\{[F]: F \in \Delta_{i}\right\}$. We denote the simplicial boundary and coboundary maps respectively by

$$
\begin{aligned}
& \partial_{\Delta, i}: C_{i}(\Delta) \rightarrow C_{i-1}(\Delta), \\
& \partial_{\Delta, i}^{*}: C_{i-1}(\Delta) \rightarrow C_{i}(\Delta),
\end{aligned}
$$

where we have identified cochains with chains via the natural inner product. We will abbreviate the subscripts in the notation for boundaries and coboundaries whenever no ambiguity can arise. We will often regard $\partial_{i}\left(\right.$ resp. $\left.\partial_{i}^{*}\right)$ as a matrix whose columns and rows (resp. rows and columns) are indexed by $\Delta_{i}$ and $\Delta_{i-1}$ respectively. The $i^{\text {th }}$ (reduced) homology group of $\Delta$ is $\tilde{H}_{i}(\Delta)=\operatorname{ker}\left(\partial_{i}\right) / \operatorname{im}\left(\partial_{i+1}\right)$, and the $i^{\text {th }}$ (reduced) Betti number $\tilde{\beta}_{i}(\Delta)$ is the rank of the largest free $R$-module summand of $\tilde{H}_{i}(\Delta)$.

2.2. Combinatorial Laplacians. We adopt the notation of [13] for the Laplacian operators (or, equivalently, matrices) of a simplicial complex. We summarize the notation and mention some fundamental identities here.

We will often work with multisets (of eigenvalues or of vertices), in which each element occurs with some nonnegative integer multiplicity. For brevity, we drop curly braces and commas when working with multisets of integers: for instance, 5553 denotes the multiset in which 5 occurs with multiplicity three and 3 occurs with multiplicity one. The cardinality of a multiset is the sum of the multiplicities of its elements; thus $|5553|=4$. We write $\mathbf{a} \stackrel{\circ}{=} \mathbf{b}$ to mean that the multisets $\mathbf{a}$ and $\mathbf{b}$ differ only in their respective multiplicities of zero; for instance, $5553 \stackrel{\circ}{=} 55530 \stackrel{\circ}{=} 555300$. Of course, $\stackrel{\circ}{=}$ is an equivalence relation. The union operation $\cup$ on multisets is understood to add multiplicities: for instance, $5553 \cup 5332=55553332$.

For $-1 \leq i \leq \operatorname{dim} \Delta$, define linear operators $L_{\Delta, i}^{\mathrm{ud}}, L_{\Delta, i}^{\mathrm{du}}, L_{\Delta, i}^{\mathrm{tot}}$ on the vector space $C_{i}(\Delta)$ by

$$
\begin{array}{ll}
L_{\Delta, i}^{\mathrm{ud}}=\partial_{i+1} \partial_{i+1}^{*} & \text { (the up-down Laplacian) }, \\
L_{\Delta, i}^{\mathrm{du}}=\partial_{i}^{*} \partial_{i} & \text { (the down-up Laplacian) }, \\
L_{\Delta, i}^{\mathrm{tot}}=L_{\Delta, i}^{\mathrm{ud}}+L_{\Delta, i}^{\mathrm{du}} & \text { (the total Laplacian) } .
\end{array}
$$

The spectrum $s_{i}^{\text {tot }}(\Delta)$ of $L_{\Delta, i}^{\text {tot }}$ is the multiset of its eigenvalues (including zero); we define $s_{i}^{\text {ud }}(\Delta)$ and $s_{i}^{\text {du }}(\Delta)$ similarly. Since each Laplacian operator is represented by a symmetric matrix, it is diagonalizable, so

$$
\left|s_{i}^{\text {tot }}(\Delta)\right|=\left|s_{i}^{\mathrm{ud}}(\Delta)\right|=\left|s_{i}^{\mathrm{du}}(\Delta)\right|=f_{i}(\Delta) .
$$


The various Laplacian spectra are related by the identities

$$
\begin{aligned}
& s_{i}^{\mathrm{ud}}(\Delta) \stackrel{\circ}{=} s_{i+1}^{\mathrm{du}}(\Delta), \\
& s_{i}^{\mathrm{tot}}(\Delta) \stackrel{\circ}{=} s_{i}^{\mathrm{ud}}(\Delta) \cup s_{i}^{\mathrm{du}}(\Delta)
\end{aligned}
$$

[13, eqn. (3.6)]. Therefore, each of the three families of multisets

$$
\begin{gathered}
\left\{s_{i}^{\mathrm{tot}}(\Delta):-1 \leq i \leq \operatorname{dim} \Delta\right\}, \quad\left\{s_{i}^{\mathrm{ud}}(\Delta):-1 \leq i \leq \operatorname{dim} \Delta\right\}, \\
\left\{s_{i}^{\mathrm{du}}(\Delta):-1 \leq i \leq \operatorname{dim} \Delta\right\}
\end{gathered}
$$

determines the other two, and we will feel free to work with whichever one is most convenient in context.

Combinatorial Laplacians and their spectra have been investigated for a number of classes of simplicial complexes. In particular, it is known that chessboard [17], matching [1], matroid [24], and shifted [13. complexes are Laplacian integral, i.e., all their Laplacian eigenvalues are integers. Understanding which complexes are Laplacian integral is an open question. As we will see, Laplacian eigenvalues and spanning tree enumerators are inextricably linked.

\section{Simplicial SPANNing TREES}

In this section, we generalize the notion of a spanning tree to arbitrary dimension using simplicial homology, following Kalai's idea. Our definition makes sense for any ambient complex that satisfies the relatively mild APC condition.

Definition 3.1. Let $\Delta^{d}$ be a simplicial complex, and let $k \leq d$. A $k$-dimensional simplicial spanning tree (for short, $k$-SST) of $\Delta$ is a $k$-dimensional subcomplex $\Upsilon \subseteq \Delta$ such that $\Upsilon_{(k-1)}=\Delta_{(k-1)}$ and

$$
\begin{aligned}
& \tilde{H}_{k}(\Upsilon)=0, \\
& \left|\tilde{H}_{k-1}(\Upsilon)\right|<\infty, \quad \text { and } \\
& f_{k}(\Upsilon)=f_{k}(\Delta)-\tilde{\beta}_{k}(\Delta)+\tilde{\beta}_{k-1}(\Delta)
\end{aligned}
$$

We write $\mathcal{T}_{k}(\Delta)$ for the set of all $k$-SST's of $\Delta^{d}$, omitting the subscript if $k=d$. Note that $\mathcal{T}_{k}(\Delta)=\mathcal{T}_{k}\left(\Delta_{(j)}\right)$ for all $j \geq k$.

A zero-dimensional SST is just a vertex of $\Delta$. If $\Delta$ is a 1-dimensional simplicial complex on $n$ vertices (that is, a graph), then the definition of 1-SST coincides with the usual definition of a spanning tree of a graph: namely, a subgraph of $\Delta$ which is connected, acyclic, and has $n-1$ edges. Next, we give a few examples in higher dimensions.

Example 3.2. If $\Delta^{d}$ is a simplicial sphere (for instance, the boundary of a simplicial polytope), then deleting any facet of $\Delta$ while keeping its $(d-1)$-skeleton intact produces a $d$-SST. Therefore $|\mathcal{T}(\Delta)|=f_{d}(\Delta)$.

Example 3.3. In dimension $>1$, spanning trees need not be $\mathbb{Z}$-acyclic, merely $\mathbb{Q}$ acyclic. For example, let $\Delta$ be a triangulation of the real projective plane, so that $\operatorname{dim} \Delta=2, \tilde{H}_{1}(\Delta, \mathbb{Z}) \cong \mathbb{Z} / 2 \mathbb{Z}$, and $\tilde{H}_{1}(\Delta, \mathbb{Q})=0$. Then $\Delta$ satisfies the conditions of Definition 3.1 and is a 2-SST of itself (in fact, the only such). 
Example 3.4. Consider the equatorial bipyramid $B$ of Example 1.7 A 2-SST of $B$ can be constructed by removing two facets $F, F^{\prime}$, provided that $F \cap F^{\prime}$ contains neither of the vertices 4,5 . A simple count shows that there are 15 such pairs $F, F^{\prime}$, so $\left|\mathcal{T}_{2}(B)\right|=15$.

Before proceeding any further, we show that Definition 3.1 satisfies a "two-outof-three theorem" akin to that for spanning trees of graphs.

Proposition 3.5. Let $\Upsilon \subset \Delta^{d}$ be a k-dimensional subcomplex with $\Upsilon_{(k-1)}=$ $\Delta_{(k-1)}$. Then any two of the conditions (6a), (6b), (6c) together imply the third.

Proof. First, note that

$$
f_{\ell}(\Upsilon)=f_{\ell}(\Delta) \quad \text { for } \ell \leq k-1 \quad \text { and } \quad \tilde{\beta}_{\ell}(\Upsilon)=\tilde{\beta}_{\ell}(\Delta) \text { for } \ell \leq k-2 .
$$

Next, we use the standard fact that the Euler characteristic $\chi(\Upsilon)$ can be calculated as the alternating sum either of the $f$-numbers or of the Betti numbers. Thus

$$
\begin{aligned}
\chi(\Upsilon) & =\sum_{i=0}^{k}(-1)^{i} f_{i}(\Upsilon) \\
& =(-1)^{k} f_{k}(\Upsilon)+\sum_{i=0}^{k-1}(-1)^{i} f_{i}(\Delta) \\
& =(-1)^{k} f_{k}(\Upsilon)+\chi(\Delta)-(-1)^{k} f_{k}(\Delta)
\end{aligned}
$$

and, on the other hand,

$$
\begin{aligned}
\chi(\Upsilon) & =\sum_{i=0}^{k}(-1)^{i} \tilde{\beta}_{i}(\Upsilon) \\
& =(-1)^{k}\left(\tilde{\beta}_{k}(\Upsilon)-\tilde{\beta}_{k-1}(\Upsilon)\right)+\sum_{i=0}^{k-2}(-1)^{i} \tilde{\beta}_{i}(\Delta) \\
& =(-1)^{k}\left(\tilde{\beta}_{k}(\Upsilon)-\tilde{\beta}_{k-1}(\Upsilon)\right)+\chi(\Delta)-(-1)^{k}\left(\tilde{\beta}_{k}(\Delta)-\tilde{\beta}_{k-1}(\Delta)\right) .
\end{aligned}
$$

Equating (8) and (91) gives

$$
f_{k}(\Upsilon)-f_{k}(\Delta)=\tilde{\beta}_{k}(\Upsilon)-\tilde{\beta}_{k-1}(\Upsilon)-\tilde{\beta}_{k}(\Delta)+\tilde{\beta}_{k-1}(\Delta)
$$

or equivalently

$$
\left(f_{k}(\Upsilon)-f_{k}(\Delta)+\tilde{\beta}_{k}(\Delta)-\tilde{\beta}_{k-1}(\Delta)\right)-\tilde{\beta}_{k}(\Upsilon)+\tilde{\beta}_{k-1}(\Upsilon)=0
$$

Since (6a) says that $\tilde{\beta}_{k}(\Upsilon)=0$ (note that $\tilde{H}_{k}(\Upsilon)$ must be free abelian) and (6b) says that $\tilde{\beta}_{k-1}(\Upsilon)=0$, the conclusion follows.

Definition 3.6. A simplicial complex $\Delta^{d}$ is acyclic in positive codimension, or APC for short, if $\tilde{\beta}_{j}(\Delta)=0$ for all $j<d$.

Equivalently, a complex $\Delta^{d}$ is APC if it has the homology type of a wedge of zero or more $d$-dimensional spheres. In particular, any Cohen-Macaulay complex is APC. The converse is very far from true, because, for instance, an APC complex need not even be pure. For our purposes, the APC complexes are the "correct" simplicial analogues of connected graphs for the following reason. 
Proposition 3.7. For any simplicial complex $\Delta^{d}$, the following are equivalent:

(1) $\triangle$ is $A P C$;

(2) $\Delta$ has a d-dimensional spanning tree;

(3) $\Delta$ has a $k$-dimensional spanning tree for every $k \leq d$.

Proof. It is trivial that (3) implies (2). To see that (2) implies (1), suppose that $\Delta$ has a $d$-dimensional spanning tree $\Upsilon$. Then $\Upsilon_{i}=\Delta_{i}$ for all $i \leq d-1$, so $\tilde{H}_{i}(\Delta)=\tilde{H}_{i}(\Upsilon)=0$ for all $i \leq d-2$. Moreover, in the diagram

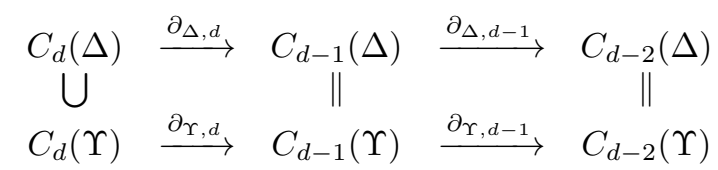

we have $\operatorname{ker} \partial_{\Delta, d-1}=\operatorname{ker} \partial_{\Upsilon, d-1}$ and $\operatorname{im} \partial_{\Delta, d} \supseteq \operatorname{im} \partial_{\Upsilon, d}$, so there is a surjection $0=\tilde{H}_{d-1}(\Upsilon) \rightarrow \tilde{H}_{d-1}(\Delta)$, implying that $\Delta$ is APC.

To prove (1) implies (3), it suffices to consider the case $k=d$, because any skeleton of an APC complex is also APC. We can construct a $d$-SST $\Upsilon$ by the following algorithm. Let $\Upsilon=\Delta$. If $\tilde{H}_{d}(\Upsilon) \neq 0$, then there exists some nonzero linear combination of facets of $\Upsilon$ that is mapped to zero by $\partial_{\Upsilon, d}$. Let $F$ be one of those facets, and let $\Upsilon^{\prime}=\Upsilon \backslash\{F\}$. Then $\tilde{\beta}_{d}\left(\Upsilon^{\prime}\right)=\tilde{\beta}_{d}(\Upsilon)-1$ and $\tilde{\beta}_{i}\left(\Upsilon^{\prime}\right)=\tilde{\beta}_{i}(\Upsilon)$ for $i \leq d-2$, and by the Euler characteristic formula, we have $\tilde{\beta}_{d-1}\left(\Upsilon^{\prime}\right)=\tilde{\beta}_{d-1}(\Upsilon)$ as well. Replacing $\Upsilon$ with $\Upsilon^{\prime}$ and repeating, we eventually arrive at the case $\tilde{H}_{d}(\Upsilon)=0$, when $\Upsilon$ is a $d$-SST of $\Delta$.

The APC condition is a fairly mild one. For instance, any $\mathbb{Q}$-acyclic complex is clearly APC (and is its own unique SST), as is any Cohen-Macaulay complex (in particular, any shifted complex).

\section{Simplicial analogues of the Matrix-Tree Theorem}

We now explain how to enumerate simplicial spanning trees of a complex using its Laplacian. Throughout this section, let $\Delta^{d}$ be an APC simplicial complex on the vertex set $[n]$. For $k \leq d$, define

$$
\pi_{k}=\pi_{k}(\Delta)=\prod_{0 \neq \lambda \in s_{k-1}^{\mathrm{ud}}(\Delta)} \lambda, \quad \tau_{k}=\tau_{k}(\Delta)=\sum_{\Upsilon \in \mathcal{T}_{k}(\Delta)}\left|\tilde{H}_{k-1}(\Upsilon)\right|^{2} .
$$

We are interested in the relationships between these two families of invariants. When $d=1$, the relationship is given by Theorem 1.1. In the notation just defined, part (1) of that theorem says that $\tau_{1}=\pi_{1} / n$, and part (2) says that $\tau_{1}=\operatorname{det} L_{i}$ (i.e., the determinant of the reduced Laplacian obtained from $L_{\Delta, 0}^{\mathrm{ud}}$ by deleting the row and column corresponding to any vertex $i$ ).

The results of this section generalize both parts of the Matrix-Tree Theorem from graphs to all APC complexes $\Delta^{d}$. Our arguments are closely based on those used by Kalai [20] and Adin [1] to enumerate SST's of skeletons of simplices and of complete colorful complexes.

We begin by setting up some notation. Abbreviate $\tilde{\beta}_{i}=\tilde{\beta}_{i}(\Delta), f_{i}=f_{i}(\Delta)$, and $\partial=\partial_{\Delta, d}$. Let $T$ be a set of facets of $\Delta$ of cardinality $f_{d}-\tilde{\beta}_{d}+\tilde{\beta}_{d-1}=f_{d}-\tilde{\beta}_{d}$, and let $S$ be a set of ridges such that $|S|=|T|$. Define

$$
\Delta_{T}=T \cup \Delta_{(d-1)}, \quad \bar{S}=\Delta_{(d-1)} \backslash S, \quad \Delta_{\bar{S}}=\bar{S} \cup \Delta_{(d-2)},
$$


and let $\partial_{S, T}$ be the square submatrix of $\partial$ with rows indexed by $S$ and columns indexed by $T$.

Proposition 4.1. The matrix $\partial_{S, T}$ is nonsingular if and only if $\Delta_{T} \in \mathcal{T}_{d}(\Delta)$ and $\Delta_{\bar{S}} \in \mathcal{T}_{d-1}(\Delta)$.

Proof. We may regard $\partial_{S, T}$ as the top boundary map of the $d$-dimensional relative complex $\Gamma=\left(\Delta_{T}, \Delta_{\bar{S}}\right)$. So $\partial_{S, T}$ is nonsingular if and only if $\tilde{H}_{d}(\Gamma)=0$. Consider the long exact sequence

$0 \rightarrow \tilde{H}_{d}\left(\Delta_{\bar{S}}\right) \rightarrow \tilde{H}_{d}\left(\Delta_{T}\right) \rightarrow \tilde{H}_{d}(\Gamma) \rightarrow \tilde{H}_{d-1}\left(\Delta_{\bar{S}}\right) \rightarrow \tilde{H}_{d-1}\left(\Delta_{T}\right) \rightarrow \tilde{H}_{d-1}(\Gamma) \rightarrow \cdots$.

If $\tilde{H}_{d}(\Gamma) \neq 0$, then $\tilde{H}_{d}\left(\Delta_{T}\right)$ and $\tilde{H}_{d-1}\left(\Delta_{\bar{S}}\right)$ cannot both be zero. This proves the "only if" direction.

If $\tilde{H}_{d}(\Gamma)=0$, then $\tilde{H}_{d}\left(\Delta_{\bar{S}}\right)=0\left(\right.$ since $\left.\operatorname{dim} \Delta_{\bar{S}}=d-1\right)$, so (10) implies $\tilde{H}_{d}\left(\Delta_{T}\right)=$ 0 . Therefore $\Delta_{T}$ is a $d$-tree, because it has the correct number of facets. Hence $\tilde{H}_{d-1}\left(\Delta_{T}\right)$ is finite. Then (10) implies that $\tilde{H}_{d-1}\left(\Delta_{\bar{S}}\right)$ is finite. In fact, it is zero because the top homology group of any complex must be torsion-free. Meanwhile, $\Delta_{\bar{S}}$ has the correct number of facets to be a $(d-1)$-SST of $\Delta$, proving the "if" direction.

Proposition 4.2. If $\partial_{S, T}$ is nonsingular, then

$$
\left|\operatorname{det} \partial_{S, T}\right|=\frac{\left|\tilde{H}_{d-1}\left(\Delta_{T}\right)\right| \cdot\left|\tilde{H}_{d-2}\left(\Delta_{\bar{S}}\right)\right|}{\left|\tilde{H}_{d-2}\left(\Delta_{T}\right)\right|}=\frac{\left|\tilde{H}_{d-1}\left(\Delta_{T}\right)\right| \cdot\left|\tilde{H}_{d-2}\left(\Delta_{\bar{S}}\right)\right|}{\left|\tilde{H}_{d-2}(\Delta)\right|} .
$$

Proof. As before, we interpret $\partial_{S, T}$ as the boundary map of the relative complex $\Gamma=\left(\Delta_{T}, \Delta_{\bar{S}}\right)$. So $\partial_{S, T}$ is a map from $\mathbb{Z}^{|T|}$ to $\mathbb{Z}^{|T|}$, and $\mathbb{Z}^{|T|} / \partial_{S, T}\left(\mathbb{Z}^{|T|}\right)$ is a finite abelian group of order $\left|\operatorname{det} \partial_{S, T}\right|$. On the other hand, since $\Gamma$ has no faces of dimension $\leq d-2$, its lower boundary maps are all zero, so $\left|\operatorname{det} \partial_{S, T}\right|=\left|\tilde{H}_{d-1}(\Gamma)\right|$. Since $\tilde{H}_{d-2}\left(\Delta_{T}\right)$ is finite, the desired result now follows from the piece

$$
0 \rightarrow \tilde{H}_{d-1}\left(\Delta_{T}\right) \rightarrow \tilde{H}_{d-1}(\Gamma) \rightarrow \tilde{H}_{d-2}\left(\Delta_{\bar{S}}\right) \rightarrow \tilde{H}_{d-2}\left(\Delta_{T}\right) \rightarrow 0
$$

of the long exact sequence (10).

We can now prove the first version of the Simplicial Matrix-Tree Theorem, relating the quantities $\pi_{d}$ and $\tau_{d}$. Abbreviate $L=L_{\Delta, d-1}^{\mathrm{ud}}$.

Theorem 1.3 (Simplicial Matrix-Tree Theorem). Let $\Delta^{d}$ be an APC simplicial complex. Then:

(1) We have

$$
\pi_{d}(\Delta)=\frac{\tau_{d}(\Delta) \tau_{d-1}(\Delta)}{\left|\tilde{H}_{d-2}(\Delta)\right|^{2}}
$$

(2) Let $U$ be the set of facets of a $(d-1)-S S T$ of $\Delta$, and let $L_{U}$ denote the reduced Laplacian 3 obtained by deleting the rows and columns of $L$ corresponding to $U$. Then

$$
\tau_{d}(\Delta)=\frac{\left|\tilde{H}_{d-2}(\Delta)\right|^{2}}{\left|\tilde{H}_{d-2}\left(\Delta_{U}\right)\right|^{2}} \operatorname{det} L_{U}
$$

\footnotetext{
${ }^{3}$ A warning: This notation for reduced Laplacians specifies which rows and columns to exclude (in analogy to the notation $L_{i}$ in the statement of Theorem 1.1), in contrast to the notation $\partial_{S, T}$ for restricted boundary maps, which specifies which rows and columns to include.
} 
Proof of Theorem 1.3 (1). The Laplacian $L$ is a square matrix with $f_{d-1}$ rows and columns, and rank $f_{d}-\tilde{\beta}_{d}=f_{d}-\tilde{\beta}_{d}+\tilde{\beta}_{d-1}$ (because $\Delta$ is APC). Let $\chi(L ; y)=$ $\operatorname{det}(y I-L)$ be its characteristic polynomial (where $I$ is an identity matrix), so that $\pi_{d}(\Delta)$, the product of the nonzero eigenvalues of $L$, is given (up to sign) by the coefficient of $y^{f_{d-1}-f_{d}+\tilde{\beta}_{d}}$ in $\chi(L ; y)$. Equivalently,

$$
\pi_{d}=\sum_{\substack{S \subset \Delta_{d-1} \\|S|=\operatorname{rank} L}} \operatorname{det} L_{U}=\sum_{\substack{S \subset \Delta_{d-1} \\|S|=f_{d}-\tilde{\beta}_{d}}} \operatorname{det} L_{U},
$$

where $U=\Delta_{d-1} \backslash S$ in each summand. By the Binet-Cauchy formula, we have

$$
\operatorname{det} L_{U}=\sum_{\substack{T \subset \Delta_{d} \\|T|=|S|}}\left(\operatorname{det} \partial_{S, T}\right)\left(\operatorname{det} \partial_{S, T}^{*}\right)=\sum_{\substack{T \subset \Delta_{d} \\|T|=|S|}}\left(\operatorname{det} \partial_{S, T}\right)^{2} .
$$

Combining (12) and (13), applying Proposition 4.1, and interchanging the sums, we obtain

$$
\pi_{d}=\sum_{T: \Delta_{T} \in \mathcal{T}_{d}(\Delta)} \sum_{S: \Delta_{\bar{S}} \in \mathcal{T}_{d-1}(\Delta)}\left(\operatorname{det} \partial_{S, T}\right)^{2}
$$

and now applying Proposition 4.2 yields

$$
\begin{aligned}
\pi_{d} & =\sum_{T: \Delta_{T} \in \mathcal{T}_{d}(\Delta)} \sum_{S: \Delta_{\bar{S}} \in \mathcal{T}_{d-1}(\Delta)}\left(\frac{\left|\tilde{H}_{d-1}\left(\Delta_{T}\right)\right| \cdot\left|\tilde{H}_{d-2}\left(\Delta_{\bar{S}}\right)\right|}{\left|\tilde{H}_{d-2}(\Delta)\right|}\right)^{2} \\
& =\frac{\left(\sum_{T: \Delta_{T} \in \mathcal{T}_{d}(\Delta)}\left|\tilde{H}_{d-1}\left(\Delta_{T}\right)\right|^{2}\right)\left(\sum_{S: \Delta_{\bar{S}} \in \mathcal{T}_{d-1}(\Delta)}\left|\tilde{H}_{d-2}\left(\Delta_{\bar{S}}\right)\right|\right)}{\left|\tilde{H}_{d-2}(\Delta)\right|^{2}}
\end{aligned}
$$

as desired.

In order to prove the "reduced Laplacian" part of Theorem 1.3. we first check that when we delete the rows of $\partial$ corresponding to a $(d-1)$-SST, the resulting reduced Laplacian has the correct size, namely, that of a $d$-SST.

Lemma 4.3. Let $U$ be the set of facets of a $(d-1)$-SST of $\Delta$, and let $S=\Delta_{d-1} \backslash U$. Then $|S|=f_{d}(\Delta)-\tilde{\beta}_{d}(\Delta)$, the number of facets of a d-SST of $\Delta$.

Proof. Let $\Gamma=\Delta_{(d-1)}$. By Proposition 3.5 and the observation (7), $|U|=f_{d-1}(\Gamma)-$ $\tilde{\beta}_{d-1}(\Gamma)+\tilde{\beta}_{d-2}(\Gamma)=f_{d-1}(\Delta)-\tilde{\beta}_{d-1}(\Gamma)$, so $|S|=\tilde{\beta}_{d-1}(\Gamma)$. The Euler characteristics of $\Delta$ and $\Gamma$ are

$$
\begin{aligned}
\chi(\Delta) & =\sum_{i=0}^{d}(-1)^{i} f_{i}(\Delta)=\sum_{i=0}^{d}(-1)^{i} \tilde{\beta}_{i}(\Delta), \\
\chi(\Gamma) & =\sum_{i=0}^{d-1}(-1)^{i} f_{i}(\Gamma)=\sum_{i=0}^{d-1}(-1)^{i} \tilde{\beta}_{i}(\Gamma) .
\end{aligned}
$$

By (7), we see that

$$
\chi(\Delta)-\chi(\Gamma)=(-1)^{d} f_{d}(\Delta)=(-1)^{d} \tilde{\beta}_{d}(\Delta)+(-1)^{d-1} \tilde{\beta}_{d-1}(\Delta)-(-1)^{d-1} \tilde{\beta}_{d-1}(\Gamma),
$$


from which we obtain $f_{d}(\Delta)=\tilde{\beta}_{d}(\Delta)-\tilde{\beta}_{d-1}(\Delta)+\tilde{\beta}_{d-1}(\Gamma)$. Since $\Delta$ is APC, we have $\tilde{\beta}_{d-1}(\Delta)=0$, so $|S|=\tilde{\beta}_{d-1}(\Gamma)=f_{d}(\Delta)-\tilde{\beta}_{d}(\Delta)$ as desired.

Proof of Theorem 1.3 (2). By the Binet-Cauchy formula, we have

$$
\operatorname{det} L_{U}=\sum_{T:|T|=|S|}\left(\operatorname{det} \partial_{S, T}\right)\left(\operatorname{det} \partial_{S, T}^{*}\right)=\sum_{T:|T|=|S|}\left(\operatorname{det} \partial_{S, T}\right)^{2} .
$$

By Lemma 4.3 and Proposition 4.1, $\partial_{S, T}$ is nonsingular exactly when $\Delta_{T} \in$ $\mathcal{T}_{d}(\Delta)$. Hence Proposition 4.2 gives

$$
\begin{aligned}
\operatorname{det} L_{U} & =\sum_{T: \Delta_{T} \in \mathcal{T}_{d}(\Delta)}\left(\frac{\left|\tilde{H}_{d-1}\left(\Delta_{T}\right)\right| \cdot\left|\tilde{H}_{d-2}\left(\Delta_{U}\right)\right|}{\left|\tilde{H}_{d-2}(\Delta)\right|}\right)^{2} \\
& =\frac{\left|\tilde{H}_{d-2}\left(\Delta_{U}\right)\right|^{2}}{\left|\tilde{H}_{d-2}(\Delta)\right|^{2}} \sum_{T: \Delta_{T} \in \mathcal{T}_{d}(\Delta)}\left|\tilde{H}_{d-1}\left(\Delta_{T}\right)\right|^{2}=\frac{\left|\tilde{H}_{d-2}\left(\Delta_{U}\right)\right|^{2}}{\left|\tilde{H}_{d-2}(\Delta)\right|^{2}} \tau_{d}(\Delta),
\end{aligned}
$$

which is equivalent to the desired formula.

Remark 4.4. Suppose that $\tilde{H}_{d-2}(\Delta)=0$ (for example, if $\Delta$ is Cohen-Macaulay). Then the two versions of Theorem 1.3 assert that

$$
\tau_{d}=\frac{\pi_{d}}{\tau_{d-1}}=\frac{\operatorname{det} L_{U}}{\left|\tilde{H}_{d-2}\left(\Delta_{U}\right)\right|^{2}},
$$

from which it is easy to recognize the two different versions of the classical MatrixTree Theorem, Theorem 1.1] (A graph is Cohen-Macaulay as a simplicial complex if and only if it is connected.) Moreover, the recurrence $\tau_{d}=\pi_{d} / \tau_{d-1}$ leads to an expression for $\tau_{d}$ as an alternating product of eigenvalues:

$$
\tau_{d}=\frac{\pi_{d} \pi_{d-2} \cdots}{\pi_{d-1} \pi_{d-3} \cdots}=\prod_{k=0}^{d} \pi_{k}^{(-1)^{d-k}} .
$$

This formula is reminiscent of the Reidemeister torsion of a chain complex or CWcomplex (although $\tau_{d}$ is of course not a topological invariant); see, e.g., 35. Furthermore, (14) is in practice an efficient way to calculate $\tau_{d}$.

Example 4.5. For the equatorial bipyramid $B$, we have

$$
\pi_{0}(B)=5, \quad \pi_{1}(B)=5 \cdot 5 \cdot 5 \cdot 3=375, \quad \pi_{2}(B)=5 \cdot 5 \cdot 5 \cdot 3 \cdot 3=1125 .
$$

These numbers can be checked by computation and also follow from the DuvalReiner formula for Laplacian eigenvalues of a shifted complex. Applying the alternating product formula (14) yields

$$
\tau_{0}(B)=5, \quad \tau_{1}(B)=375 / 5=75, \quad \tau_{2}(B)=\frac{1125 \cdot 5}{375}=15 .
$$

Indeed, $\tau_{0}(B)$ is the number of vertices. Cayley's formula implies that deleting any one edge $e$ from $K_{n}$ yields a graph with $(n-2) n^{n-3}$ spanning trees (because $e$ itself belongs to $(n-1) /\left(\begin{array}{l}n \\ 2\end{array}\right)$ of the spanning trees of $\left.K_{n}\right)$, and the 1-skeleton $B_{(1)}$ is such a graph with $n=5$, so $\tau_{1}(B)=75$. Finally, we have seen in Example 3.4 that $\tau_{2}(B)=15$. 


\section{Weighted ENUMERATION OF SIMPLICIAL SPANNING TREES}

We can obtain much finer enumerative information by labeling the facets of a complex with indeterminates, so that the invariant $\tau_{k}$ becomes a generating function for its SST's.

Let $\Delta^{d}$ be an APC simplicial complex, and let $\partial=\partial_{\Delta, d}$. Introduce an indeterminate $x_{F}$ for each facet $F$ of maximum dimension, and let $X_{F}=x_{F}^{2}$. For every $T \subseteq \Delta_{d}$, let $x_{T}=\prod_{F \in T} x_{F}$ and let $X_{T}=x_{T}^{2}$. To construct the weighted boundary matrix $\hat{\partial}$ from $\partial$, multiply each column of $\hat{\partial}$ by $x_{F}$, where $F$ is the facet of $\Delta$ corresponding to that column. The weighted coboundary $\hat{\partial}^{*}$ is the transpose of $\hat{\partial}$. We can now define weighted versions of Laplacians, the various submatrices of the boundary and coboundary matrices used in Section 4, and the invariants $\pi_{k}$ and $\tau_{k}$. We will notate each weighted invariant by placing a hat over the symbol for the corresponding unweighted quantity. Thus $\hat{\pi}_{k}$ is the product of the nonzero eigenvalues of $\hat{L}_{\Delta, k-1}^{\mathrm{ud}}$, and

$$
\hat{\tau}_{k}=\hat{\tau}_{k}(\Delta)=\sum_{\Upsilon \in \mathcal{T}_{k}(\Delta)}\left|\tilde{H}_{k-1}(\Upsilon)\right|^{2} X_{\Upsilon}
$$

To recover any unweighted quantity from its weighted analogue, set $x_{F}=1$ for all $F \in \Delta_{d}$.

Proposition 5.1. Let $T \subset \Delta_{d}$ and $S \subset \Delta_{d-1}$, with $|T|=|S|=f_{d}-\tilde{\beta}_{d}$. Then $\operatorname{det} \hat{\partial}_{S, T}=x_{T} \operatorname{det} \partial_{S, T}$ is nonzero if and only if $\Delta_{T} \in \mathcal{T}_{d}(\Delta)$ and $\Delta_{\bar{S}} \in \mathcal{T}_{d-1}(\Delta)$. In that case,

$$
\pm \operatorname{det} \hat{\partial}_{S, T}=\frac{\left|\tilde{H}_{d-1}\left(\Delta_{T}\right)\right| \cdot\left|\tilde{H}_{d-2}\left(\Delta_{\bar{S}}\right)\right|}{\left|\tilde{H}_{d-2}\left(\Delta_{T}\right)\right|} x_{T}=\frac{\left|\tilde{H}_{d-1}\left(\Delta_{T}\right)\right| \cdot\left|\tilde{H}_{d-2}\left(\Delta_{\bar{S}}\right)\right|}{\left|\tilde{H}_{d-2}(\Delta)\right|} x_{T}
$$

Proof. The first claim follows from Proposition 4.1, and the second follows from Proposition 4.2 .

It is now straightforward to adapt the proofs of both parts of Theorem 1.3 to the weighted setting. For convenience, we restate the result. Let $\hat{L}=\hat{L}_{\Delta, d-1}^{\mathrm{ud}}$.

Theorem 1.4 (Weighted Simplicial Matrix-Tree Theorem). Let $\Delta^{d}$ be an APC simplicial complex. Then:

(1) We have

$$
\hat{\pi}_{d}(\Delta)=\frac{\hat{\tau}_{d}(\Delta) \tau_{d-1}(\Delta)}{\left|\tilde{H}_{d-2}(\Delta)\right|^{2}}
$$

(2) Let $U$ be the set of facets of a $(d-1)$-SST of $\Delta$, and let $\hat{L}_{U}$ be the reduced Laplacian obtained by deleting the rows and columns of $\hat{L}$ corresponding to $U$. Then

$$
\hat{\tau}_{d}(\Delta)=\frac{\left|\tilde{H}_{d-2}(\Delta)\right|^{2}}{\left|\tilde{H}_{d-2}\left(\Delta_{U}\right)\right|^{2}} \operatorname{det} \hat{L}_{U}
$$


Proof. For assertion (1), we use a weighted version of the argument of part (1) of Theorem 1.3. By the Binet-Cauchy formula and Proposition 5.1, we have

$$
\begin{aligned}
\hat{\pi}_{d} & =\sum_{S \subset \Delta_{d-1}} \sum_{\substack{T \subset \Delta_{d} \\
|T|=|S|}}\left(\operatorname{det} \hat{\partial}_{T, S}^{*}\right)\left(\operatorname{det} \hat{\partial}_{S, T}\right)=\sum_{S} \sum_{T}\left(\operatorname{det} \hat{\partial}_{S, T}\right)^{2} \\
& =\sum_{T: \Delta_{T} \in \mathcal{T}_{d}(\Delta)} \sum_{S: \Delta_{\bar{S}} \in \mathcal{T}_{d-1}(\Delta)}\left(\operatorname{det} \hat{\partial}_{S, T}\right)^{2} \\
& =\sum_{T: \Delta_{T} \in \mathcal{T}_{d}(\Delta)} \sum_{S: \Delta_{\bar{S}} \in \mathcal{T}_{d-1}(\Delta)}\left(\frac{\left|\tilde{H}_{d-1}\left(\Delta_{T}\right)\right| \cdot\left|\tilde{H}_{d-2}\left(\Delta_{\bar{S}}\right)\right|}{\left|\tilde{H}_{d-2}(\Delta)\right|}\right)^{2} X_{T}=\frac{\hat{\tau}_{d}(\Delta) \tau_{d-1}(\Delta)}{\left|\tilde{H}_{d-2}(\Delta)\right|^{2}} .
\end{aligned}
$$

The proof of assertion (2) of the theorem is identical to that of part (2) of Theorem 1.3, using Proposition 5.1 instead of Proposition 4.2 .

Example 5.2. We return to the equatorial bipyramid $B$ of Example 1.7. Weight each facet $F=\{i, j, k\}$ by the monomial $x_{F}=x_{i} x_{j} x_{k}$. Let $U=\{12,13,14,15\}$ be the facets of a 1-SST of $B_{(1)}$. Then the reduced Laplacian $\hat{L}_{U}$ is

$$
\left(\begin{array}{ccccc}
x_{2} x_{3}\left(x_{1}+x_{4}+x_{5}\right) & -x_{2} x_{3} x_{5} & x_{2} x_{3} x_{4} & x_{2} x_{3} x_{5} & -x_{2} x_{3} x_{4} \\
-x_{2} x_{3} x_{4} & x_{2} x_{5}\left(x_{1}+x_{3}\right) & 0 & -x_{2} x_{3} x_{5} & 0 \\
x_{2} x_{3} x_{4} & 0 & x_{3} x_{4}\left(x_{1}+x_{2}\right) & 0 & -x_{2} x_{3} x_{4} \\
x_{2} x_{3} x_{5} & -x_{2} x_{3} x_{5} & 0 & x_{3} x_{5}\left(x_{1}+x_{2}\right) & 0 \\
-x_{2} x_{3} x_{4} & 0 & -x_{2} x_{3} x_{4} & 0 & x_{2} x_{4}\left(x_{1}+x_{3}\right)
\end{array}\right)
$$

and the generating function for 2-SST's by their degree sequences is

$$
\begin{aligned}
\hat{\tau}_{2}(B) & =\operatorname{det} \hat{L}_{S} \\
& =\sum_{\Upsilon \in \mathcal{T}(B)} \prod_{i \in[5]} x_{i}^{\operatorname{deg}_{B}(i)}=x_{1}^{3} x_{2}^{3} x_{3}^{3} x_{4}^{2} x_{5}^{2}\left(x_{1}+x_{2}+x_{3}\right)\left(x_{1}+x_{2}+x_{3}+x_{4}+x_{5}\right),
\end{aligned}
$$

where $\operatorname{deg}_{B}(i)$ means the number of facets of $B$ containing vertex $i$. Setting $x_{i}=1$ for every $i$ recovers the unweighted equality $\tau_{2}(B)=15$ (see Examples 3.4 and 4.5).

\section{ShifTed COMPleXes}

6.1. General definitions. In the next several sections of the paper, we apply the tools just developed to the important class of shifted complexes. We begin by reviewing some standard facts about shifted complexes and shifted families; for more details, see, e.g., 21.

Let $k$ be an integer. A $k$-set is a set of integers of cardinality $k$. A $k$-family is a set of $k$-sets (for example, the set of $(k-1)$-dimensional faces of a simplicial complex). The componentwise partial order $\preceq$ on $k$-sets of integers is defined as follows: if $A=\left\{a_{1}<a_{2}<\cdots<a_{k}\right\}$ and $B=\left\{b_{1}<b_{2}<\cdots<b_{k}\right\}$, then $A \preceq B$ if $a_{j} \leq b_{j}$ for all $j$. A $k$-family $\mathcal{F}$ is shifted if $B \in \mathcal{F}$ and $A \preceq B$ together imply $A \in \mathcal{F}$. Equivalently, $\mathcal{F}$ is shifted if it is an order ideal with respect to the componentwise partial order. A simplicial complex $\Sigma$ is shifted if $\Sigma_{i}$ is shifted for all $i$. Accordingly, we may specify a shifted complex by the list of its facets that are maximal with respect to $\preceq$, writing $\Sigma=\left\langle F_{1}, \ldots, F_{n}\right\rangle$. For example, the bipyramid of Example 1.7 is the shifted complex $\langle 235\rangle$. We will not lose any generality by assuming that the vertex set for every shifted complex we encounter is an integer 
interval $[p, q]=\{p, p+1, \ldots, q\}$; in particular, we will use the symbol $p$ throughout for the vertex with the smallest index.

The deletion and link of $\Sigma$ with respect to $p$ are defined to be the subcomplexes

$$
\begin{aligned}
\Delta & =\operatorname{del}_{p} \Sigma=\{F \backslash\{p\}: F \in \Sigma\}, \\
\Lambda & =\operatorname{link}_{p} \Sigma=\{F: p \notin F, F \cup\{p\} \in \Sigma\} .
\end{aligned}
$$

It is easy to see that the deletion and link of a shifted complex on $[p, q]$ are themselves shifted complexes 4 on $[p+1, q]$.

A complex $\Sigma$ on a vertex set $V$ is called a near-cone with apex $p$ if it has the following property: if $F \in \operatorname{del}_{p} \Sigma$ and $v \in F$, then $F \backslash\{v\} \in \operatorname{link}_{p} \Sigma$ (equivalently, $F \backslash\{v\} \cup\{p\} \in \Sigma)$. It is easy to see that a shifted complex on $[p, q]$ is a near-cone with apex $p$. Björner and Kalai [5, Theorem 4.3] showed that the Betti numbers of a shifted complex $\Sigma$ (indeed, of a near-cone) with initial vertex $p$ are given by

$$
\tilde{\beta}_{i}(\Sigma)=\left|\left\{F \in \Sigma_{i}: p \notin F, F \dot{\cup}\{p\} \notin \Sigma\right\}\right| .
$$

6.2. The combinatorial fine weighting. Let $\left\{x_{i, j}\right\}$ be a set of indeterminates, indexed by integers $i, j$. Let $\mathbb{k}$ be the field of rational functions in the $x_{i, j}$ with coefficients in $\mathbb{C}$ (or in any other field of characteristic zero). Since these indeterminates will often appear squared, we set $X_{i, j}=x_{i, j}^{2}$. The combinatorial fine weighting assigns to a multiset of vertices $S=\left\{i_{1} \leq i_{2} \leq \cdots \leq i_{m}\right\}$ the monomials

$$
x_{S}=x_{1, i_{1}} x_{2, i_{2}} \cdots x_{m, i_{m}} \text { and } X_{S}=X_{1, i_{1}} X_{2, i_{2}} \cdots X_{m, i_{m}} .
$$

Our goal is to describe the generating function

$$
\hat{\tau}_{d}(\Sigma)=\sum_{\Upsilon \in \mathcal{T}(\Sigma)}\left|\tilde{H}_{d-1}(\Sigma, \mathbb{Z})\right|^{2} X_{\Upsilon}
$$

of a shifted complex $\Sigma$, where, for each simplicial spanning tree $\Upsilon$, the monomial

$$
X_{\Upsilon}=\prod_{\text {facets } F \in \Upsilon} X_{F}
$$

records both the number of facets of $\Upsilon$ containing each vertex of $\Sigma$, as well as the order in which the vertices appear in facets.

Define the "raising operator" $\uparrow$ by $\uparrow x_{i, j}=x_{i+1, j}$ for $i \leq d$ and $\uparrow x_{d+1, j}=0$. We extend $\uparrow$ linearly and multiplicatively to an operator on all of $\mathbb{k}$. The raising operator can also be applied to a $\mathbb{k}$-linear operator $f$ by the rule

$$
(\uparrow f)(V)=\uparrow\left(f\left(\uparrow^{-1}(V)\right)\right)
$$

for any vector $V$ over $\mathbb{k}$. The $a^{\text {th }}$ iterate of $\uparrow$ is denoted by $\uparrow a$.

The following identities will be useful. Let $\tilde{S}=S \cup\{p\}$, where $\cup$ denotes the union as multisets, so that the multiplicity of $p$ in $\tilde{S}$ is one more than its multiplicity in $S$. Then, for all integers $a, j$,

$$
\uparrow^{a} x_{1, p} \cdot \uparrow^{a+1} x_{S \cup j}=\uparrow^{a}\left(x_{1, p} \cdot \uparrow x_{S \cup j}\right)=\uparrow^{a} x_{\tilde{S} \cup j}
$$

\footnotetext{
${ }^{4}$ This is also true for the deletion and link with respect to any vertex, not just $p$, but then the resulting vertex set is no longer a set of consecutive integers. Since we will not have any need to take the deletion and link with respect to any vertex other than $p$, we won't worry about that, and instead enjoy the resulting simplicity of specifying the new minimal vertex of the deletion and link.
} 
and

$$
\frac{\uparrow^{a} x_{\tilde{S}}}{\uparrow^{a+1} x_{S}}=x_{a+1, p}=\uparrow^{a} x_{1, p} .
$$

The same identities hold if $x$ is replaced with $X$.

Now, define the combinatorially finely weighted simplicial boundary map of $\Sigma$ as the homomorphism $\hat{\partial}=\hat{\partial}_{\Sigma, i}: C_{i}(\Sigma) \rightarrow C_{i-1}(\Sigma)$ which acts on generators $[F]$ (for $\left.F \in \Sigma_{i}\right)$ by

$$
\hat{\partial}[F]=\sum_{j \in F} \varepsilon(j, F) \uparrow^{d-i} x_{F}[F \backslash j] .
$$

Here we have set $\varepsilon(v, F)=(-1)^{j+1}$ if $v$ is the $j^{\text {th }}$ smallest vertex of $F$, and $\varepsilon(v, F)=$ 0 if $v \notin F$. Similarly define the finely weighted simplicial coboundary map $\hat{\partial}^{*}=$ $\hat{\partial}_{F, i+1}^{*}: C_{i}(\Sigma) \rightarrow C_{i+1}(\Sigma)$ by

$$
\hat{\partial}^{*}[F]=\sum_{j \in V \backslash F} \varepsilon(j, F \cup j) \uparrow^{d-i-1} x_{F \cup j}[F \cup j] .
$$

These maps do not make the chain groups of $\Sigma$ into an algebraic chain complex, because $\hat{\partial} \hat{\partial}$ and $\hat{\partial}^{*} \hat{\partial}^{*}$ do not vanish in general. (We will fix this problem in Section 6.3.) On the other hand, they have combinatorial significance, because we will be able to apply part (2) of Theorem 1.4 to the finely weighted up-down Laplacian $\hat{L}^{\mathrm{ud}}=\hat{\partial}_{d} \hat{\partial}_{d}^{*}$. This Laplacian may be regarded as a matrix whose rows and columns are indexed by $\Sigma_{d-1}$. It is not hard to check that for each $F, G \in \Sigma_{d-1}$, the corresponding entry of $\hat{L}^{\text {ud }}$ is

$$
\left(\hat{L}^{\mathrm{ud}}\right)_{F G}= \begin{cases}\varepsilon(j, H) \varepsilon(i, H) X_{H} & \text { if } H=F \cup j=G \cup i \in \Sigma, \\ \sum_{F \cup j \in \Sigma} X_{F \cup j} & \text { if } F=G, \\ 0 & \text { otherwise. }\end{cases}
$$

Let $U$ be the simplicial spanning tree of $\Sigma_{(d-1)}$ consisting of all ridges containing the vertex $p$. (This subcomplex is an SST because it has a complete $(d-2)$-skeleton and is a cone over $p$, hence contractible.) Let $\hat{L}_{U}^{\mathrm{ud}}$ be the reduced Laplacian obtained from $\hat{L}^{\mathrm{ud}}$ by deleting the corresponding rows and columns, so that the remaining rows and columns are indexed by the facets of $\Lambda=\operatorname{link}_{p} \Sigma$. Then part (2) of Theorem 1.4 asserts that $\hat{\tau}_{d}(\Sigma)=\operatorname{det} \hat{L}_{U}^{\mathrm{ud}}$.

Let $N=N(\Sigma)$ be the matrix obtained from $\hat{L}_{U}^{\mathrm{ud}}$ by dividing each row $F$ by $\uparrow x_{F}$ and dividing each column $G$ by $\uparrow x_{G}$. The $(F, G)$ entry of $N$ is thus

$$
N_{F G}= \begin{cases}\varepsilon(j, H) \varepsilon(i, H) \frac{X_{H}}{\uparrow x_{F} \uparrow x_{G}} & \text { if } H=F \cup j=G \cup i \in \Sigma, \\ \sum_{j: F \cup j \in \Sigma} \frac{X_{F \cup j}}{\uparrow X_{F}} & \text { if } F=G, \\ 0 & \text { otherwise. }\end{cases}
$$


Moreover,

$$
\hat{\tau}_{d}(\Sigma)=\operatorname{det} \hat{L}_{U}^{\mathrm{ud}}=\left(\prod_{F \in \Lambda_{d-1}} \uparrow X_{F}\right) \operatorname{det} N .
$$

We will shortly see (Lemma 6.3) that $N$ is almost identical to the (full) Laplacian of the deletion $\Delta=\operatorname{del}_{p} \Sigma$.

6.3. The algebraic fine weighting. The combinatorial fine weighting just defined is awkward to work with directly, because the simplicial boundary and coboundary maps (20) and (21) do not fit together into an algebraic chain complex. Therefore, we introduce a new weighting by Laurent monomials, the algebraic fine weighting, that does give the structure of a chain complex, behaves well with respect to cones and near-cones, and is easy to translate into the combinatorial fine weighting.

Definition 6.1. Let $\Delta^{d}$ be a simplicial complex on vertices $V \subset \mathbb{N}$. The algebraic finely weighted simplicial boundary map of $\Delta$ is the homomorphism $\partial_{\Delta, i}: C_{i}(\Delta) \rightarrow$ $C_{i-1}(\Delta)$ given by

$$
\boldsymbol{\partial}_{\Delta, i}[F]=\sum_{j \in F} \varepsilon(j, F) \frac{\uparrow^{d-i}\left(x_{F}\right)}{\uparrow^{d-i+1}\left(x_{F \backslash j}\right)}[F \backslash j]
$$

and similarly the algebraic finely weighted simplicial coboundary map $\boldsymbol{\partial}_{\Delta, i+1}^{*}$ : $C_{i}(\Delta) \rightarrow C_{i+1}(\Delta)$ is given by

$$
\boldsymbol{\partial}_{\Delta, i+1}^{*}[F]=\sum_{j \in V \backslash F} \varepsilon(j, F \cup j) \frac{\uparrow^{d-i-1}\left(x_{F \cup j}\right)}{\uparrow^{d-i}\left(x_{F}\right)}[F \cup j] .
$$

We will sometimes drop one or both subscripts when no confusion can arise. By the formula (18), we can apply the raising operator $\uparrow$ to $\boldsymbol{\partial}$ and $\boldsymbol{\partial}^{*}$ by applying it to each matrix entry. That is, for $[F] \in C_{i}(\Delta)$ and $a \in \mathbb{N}$, we have

$$
\begin{aligned}
\uparrow^{a} \boldsymbol{\partial}_{\Delta, i}[F] & =\sum_{j \in F} \varepsilon(j, F) \frac{\uparrow^{d-i+a}\left(x_{F}\right)}{\uparrow^{d-i+a+1}\left(x_{F \backslash j}\right)}[F \backslash j], \\
\uparrow^{a} \boldsymbol{\partial}_{\Delta, i}^{*}[F] & =\sum_{j \in V \backslash F} \varepsilon(j, F \cup j) \frac{\uparrow^{d-i+a-1}\left(x_{F \cup j}\right)}{\uparrow^{d-i+a}\left(x_{F}\right)}[F \cup j] .
\end{aligned}
$$

Lemma 6.2. Let $a \in \mathbb{N}$. Then $\uparrow^{a} \boldsymbol{\partial} \circ \uparrow^{a} \boldsymbol{\partial}=0$ and $\uparrow^{a} \boldsymbol{\partial}^{*} \circ \uparrow^{a} \boldsymbol{\partial}^{*}=0$. That is, the algebraic finely weighted boundary and coboundary operators induce chain complexes

$$
\begin{aligned}
& \cdots \rightarrow C_{i+1}(\Delta) \stackrel{\partial_{\Delta, i+1}}{\longrightarrow} C_{i}(\Delta) \stackrel{\partial_{\Delta, i}}{\longleftrightarrow} C_{i-1}(\Delta) \rightarrow \cdots, \\
& \cdots \leftarrow C_{i+1}(\Delta) \stackrel{\partial_{\Delta, i+1}^{*}}{\longleftarrow} C_{i}(\Delta) \stackrel{\partial_{\Delta, i}^{*}}{\longleftarrow} C_{i-1}(\Delta) \leftarrow \cdots .
\end{aligned}
$$


Proof. Since the matrices that represent the maps $\boldsymbol{\partial}$ and $\boldsymbol{\partial}^{*}$ are mutual transposes, it suffices to prove the first assertion. For $F \in \Delta_{i}$, we have by (27),

$$
\begin{aligned}
& \uparrow^{a} \boldsymbol{\partial}_{\Delta, i-1}\left(\uparrow a \partial_{\Delta, i}([F])\right)=\uparrow^{a} \boldsymbol{\partial}_{\Delta, i-1}\left(\sum_{j \in F} \varepsilon(j, F) \frac{\uparrow^{d-i+a}\left(x_{F}\right)}{\uparrow^{d-i+a+1}\left(x_{F \backslash j}\right)}[F \backslash j]\right) \\
& =\sum_{j \in F} \varepsilon(j, F) \frac{\uparrow^{d-i+a}\left(x_{F}\right)}{\uparrow^{d-i+a+1}\left(x_{F \backslash j}\right)} \cdot \uparrow^{a} \boldsymbol{\partial}_{\Delta, i-1}([F \backslash j]) \\
& =\sum_{j \in F} \varepsilon(j, F) \frac{\uparrow^{d-i+a}\left(x_{F}\right)}{\uparrow^{d-i+a+1}\left(x_{F \backslash j}\right)} \sum_{k \in F \backslash j} \varepsilon(k, F \backslash j) \frac{\uparrow^{d-i+a+1}\left(x_{F \backslash j}\right)}{\uparrow^{d-i+a+2}\left(x_{F \backslash j \backslash k}\right)}[F \backslash j \backslash k] \\
& =\sum_{j \in F} \sum_{k \in F \backslash j} \varepsilon(j, F) \varepsilon(k, F \backslash j) \frac{\uparrow^{d-i+a}\left(x_{F}\right)}{\uparrow^{d-i+a+2}\left(x_{F \backslash j \backslash k}\right)}[F \backslash j \backslash k] \\
& =\sum_{\substack{j, k \in F \\
j \neq k}}(\varepsilon(j, F) \varepsilon(k, F \backslash j)+\varepsilon(k, F) \varepsilon(j, F \backslash k)) \frac{\uparrow^{d-i+a}\left(x_{F}\right)}{\uparrow^{d-i+a+2}\left(x_{F \backslash j \backslash k}\right)}[F \backslash j \backslash k],
\end{aligned}
$$

and it is a standard fact of simplicial homology theory that the expression in parentheses is zero.

Define the algebraic finely weighted up-down, down-up, and total Laplacians by

$$
\mathbf{L}_{\Delta, i}^{\mathrm{ud}}=\boldsymbol{\partial}_{\Delta, i+1} \boldsymbol{\partial}_{\Delta, i+1}^{*}, \quad \mathbf{L}_{\Delta, i}^{\mathrm{du}}=\boldsymbol{\partial}_{\Delta, i}^{*} \boldsymbol{\partial}_{\Delta, i}, \quad \mathbf{L}_{\Delta, i}^{\mathrm{tot}}=\mathbf{L}_{\Delta, i}^{\mathrm{ud}}+\mathbf{L}_{\Delta, i}^{\mathrm{du}} .
$$

Each of these is a linear endomorphism of $C_{i}(\Delta)$, represented by a symmetric matrix, hence diagonalizable. Let $\mathbf{s}_{i}^{\mathrm{ud}}(\Delta), \mathbf{s}_{i}^{\mathrm{du}}(\Delta)$, and $\mathbf{s}_{i}^{\text {tot }}(\Delta)$ denote the spectra (multisets of eigenvalues) of $\mathbf{L}_{\Delta, i}^{\mathrm{ud}}, \mathbf{L}_{\Delta, i}^{\mathrm{du}}$, and $\mathbf{L}_{\Delta, i}^{\text {tot }}$ respectively. We will use the abbreviations $\mathbf{L}^{\text {ud }}, \mathbf{L}^{\text {du }}, \mathbf{L}^{\text {tot }}, \mathbf{s}^{\text {ud }}, \mathbf{s}^{\text {du }}, \mathbf{s}^{\text {tot }}$ when no confusion can arise.

If we regard $\mathbf{L}_{\Delta, d-1}^{\mathrm{ud}}$ as a matrix with rows and columns indexed by $\Delta_{d-1}$, then it is not hard to check that its $(F, G)$ entry is

$$
\left(\mathbf{L}_{\Delta, d-1}^{\mathrm{ud}}\right)_{F G}= \begin{cases}\varepsilon(j, H) \varepsilon(i, H) \frac{X_{H}}{\uparrow x_{F} \uparrow x_{G}} & \text { if } H=F \cup j=G \cup i \in \Delta, \\ \sum_{j: F \cup j \in \Delta} \frac{X_{F \cup j}}{\uparrow X_{F}} & \text { if } F=G, \\ 0 & \text { otherwise. }\end{cases}
$$

This matrix is almost identical to the matrix $N(\Sigma)$ defined in (23) when $\Delta=$ $\operatorname{del}_{p} \Sigma$, as we now explain.

Lemma 6.3. Let $\Sigma^{d}$ be a pure shifted complex with initial vertex $p$, and let $\Lambda=$ $\operatorname{link}_{p} \Sigma$ and $\Delta=\operatorname{del}_{p} \Sigma$. Then

$$
\hat{\tau}_{d}(\Sigma)=\left(\prod_{F \in \Lambda_{d-1}} \uparrow X_{F}\right) \prod_{\lambda \in \mathbf{s}_{\Delta, d-1}^{\mathbf{u d}}}\left(X_{1, p}+\lambda\right) .
$$

Proof. First, note that $\mathbf{L}=\mathbf{L}_{\Delta, d-1}^{\mathrm{ud}}$ is indexed by the faces of $\Delta_{d-1}$ and $N=N(\Sigma)$ is indexed by the faces of $\Lambda_{d-1}$. These indexing sets coincide because $\Sigma$ is shifted and pure of dimension $d$. 
Second, we show that the off-diagonal entries (the first cases in (23) and (29)) coincide. Suppose that $F, G$ are distinct faces in $\Delta_{d-1}=\Lambda_{d-1}$, and that $H=$ $F \cup i=G \cup j$. Suppose that $i \neq j$ and $H=F \cup i=G \cup j$. We must show that $H \in \Delta$ if and only if $H \in \Sigma$. The "only if" direction is immediate because $\Delta \subset \Sigma$. On the other hand, $H=F \cup G$ and no element of $\Delta_{d-1}$ contains $p$. Therefore, if $H \in \Sigma$, then $H \in \Delta$, as desired.

Third, we compare the entries on the main diagonals of $\mathbf{L}$ and $N$. Their only difference is that the summand with $j=p$ occurs in the second case of (23), but not in (29). Hence

$$
N_{F F}=\mathbf{L}_{F F}+\frac{X_{F \cup p}}{\uparrow X_{F}}=\mathbf{L}_{F F}+X_{1, p}
$$

by (19b). Therefore $N=\mathbf{L}+X_{1, p} I$, where $I$ is an identity matrix of size $f_{d-1}(\Delta)$, and

$$
\operatorname{det} N=\chi\left(-\mathbf{L}, X_{1, p}\right)=\prod_{\lambda \in \mathbf{s}_{\Delta, d-1}^{\text {ud }}}\left(X_{1, p}+\lambda\right)
$$

where $\chi$ denotes the characteristic polynomial of $-\mathbf{L}$ in the variable $X_{1, p}$. The lemma now follows from equation (24).

The goal of the next two sections is to compute $\mathbf{L}_{\Delta, d-1}^{\mathrm{ud}}$.

\section{CONES AND NEAR-CONES}

A shifted complex is an iterated near-cone, so we want to describe the Laplacian eigenvalues of a near-cone in terms of its base. Before we do so, we must consider the case of a cone. Proposition 7.2 provides the desired recurrence for cones, and Proposition 7.6 for near-cones.

7.1. Boundary and coboundary operators of cones. Let $\Gamma$ be the simplicial complex with the single vertex 1 , and let $\Delta^{d}$ be any complex on $V=[2, n]$. For a face $F \in \Delta$, write $\tilde{F}=1 \cup F$. The corresponding cone is

$$
\Sigma=1 * \Delta=\Gamma * \Delta=\{F, \tilde{F}: F \in \Delta\} .
$$

We will make use of the identification

$$
C_{i}(\Sigma) \cong\left(C_{-1}(\Gamma) \otimes C_{i}(\Delta)\right) \oplus\left(C_{0}(\Gamma) \otimes C_{i-1}(\Delta)\right) .
$$

By (27) and (28), the (raised) boundary and coboundary maps on $\Gamma$ are given explicitly by

$$
\begin{aligned}
\uparrow^{a} \partial_{\Gamma}[1] & =x_{a+1,1}[\emptyset], & & \uparrow^{a} \partial_{\Gamma}^{*}[1]=0, \\
\uparrow^{a} \partial_{\Gamma}[\emptyset] & =0, & \uparrow^{a} \partial_{\Gamma}^{*}[\emptyset] & =x_{a+1,1}[1] .
\end{aligned}
$$

Next, we give explicit formulas for the maps $\boldsymbol{\partial}_{\Sigma, i}$ and $\boldsymbol{\partial}_{\Sigma, i+1}^{*}$. How these maps act on a face of $\Sigma$ depends on whether it is of the form $F$, for $F \in \Delta_{i}$, or $\tilde{F}$, for $F \in \Delta_{i-1}$. Note that in any case, $\varepsilon(1, \tilde{F})=1$, and that for all $v \in F$ we have 
$\varepsilon(v, F)=-\varepsilon(v, \tilde{F})$. Therefore,

$$
\begin{aligned}
& \boldsymbol{\partial}_{\Sigma, i}([\emptyset]\otimes[F])=\sum_{j \in F} \varepsilon(j, F) \frac{\uparrow^{d-i+1}\left(x_{F}\right)}{\uparrow^{d-i+2}\left(x_{F \backslash j}\right)}[\emptyset] \otimes[F \backslash j] \\
&= \uparrow\left(\sum_{j \in F} \varepsilon(j, F) \frac{\uparrow^{d-i}\left(x_{F}\right)}{\uparrow^{d-i+1}\left(x_{F \backslash j}\right)}[\emptyset] \otimes[F \backslash j]\right) \\
& \quad=\left(\operatorname{id} \otimes \uparrow \partial_{\Delta, i}\right)([\emptyset] \otimes[F]),
\end{aligned}
$$

$$
\begin{aligned}
& \partial_{\Sigma, i}([1] \otimes[F])=\varepsilon(1, \tilde{F}) \frac{\uparrow^{d-i+1}\left(x_{\tilde{F}}\right)}{\uparrow^{d-i+2}\left(x_{F}\right)}[\emptyset] \otimes[F]+\sum_{j \in F} \varepsilon(j, \tilde{F}) \frac{\uparrow^{d-i+1}\left(x_{\tilde{F}}\right)}{\uparrow^{d-i+2}\left(x_{\tilde{F} \backslash j}\right)}[1] \otimes[F \backslash j] \\
& =x_{d-i+2,1}[\emptyset] \otimes[F]-\sum_{j \in F} \varepsilon(j, F) \frac{x_{d-i+2,1}}{x_{d-i+3,1}} \frac{\uparrow^{d-i+2}\left(x_{F}\right)}{\uparrow^{d+i+3}\left(x_{F \backslash j}\right)}[1] \otimes[F \backslash j] \\
& =x_{d-i+2,1}[\emptyset] \otimes[F]-\frac{x_{d-i+2,1}}{x_{d-i+3,1}} \uparrow\left(\sum_{j \in F} \varepsilon(j, F) \frac{\uparrow^{d-i+1}\left(x_{F}\right)}{\uparrow^{d-i+2}\left(x_{F \backslash j}\right)}[1] \otimes[F \backslash j]\right) \\
& \text { (31b) } \quad=\left(\uparrow^{d-i+1} \partial_{\Gamma} \otimes \mathrm{id}-\frac{x_{d-i+2,1}}{x_{d-i+3,1}} \mathrm{id} \otimes \uparrow \partial_{\Delta, i-1}\right)([1] \otimes[F]), \\
& \partial_{\Sigma, i+1}^{*}([\emptyset] \otimes[F])=\varepsilon(1, \tilde{F}) \frac{\uparrow^{d-i}\left(x_{\tilde{F}}\right)}{\uparrow^{d-i+1}\left(x_{F}\right)}[1] \otimes[F] \\
& +\sum_{j \in V \backslash F} \varepsilon(j, F \cup j) \frac{\uparrow^{d-i}\left(x_{F \cup j}\right)}{\uparrow^{d-i+1}\left(x_{F}\right)}[\emptyset] \otimes[F \cup j] \\
& =x_{d-i+1,1}[1] \otimes[F]+\uparrow\left(\sum_{j \in V \backslash F} \varepsilon(j, F \cup j) \frac{\uparrow^{d-i-1}\left(x_{F \cup j}\right)}{\uparrow^{d-i}\left(x_{F}\right)}[\emptyset] \otimes[F \cup j]\right) \\
& (31 \mathrm{c}) \quad=\left(\uparrow^{d-i} \boldsymbol{\partial}_{\Gamma}^{*} \otimes \mathrm{id}+\mathrm{id} \otimes \uparrow \boldsymbol{\partial}_{\Delta, i+1}^{*}\right)([\emptyset] \otimes[F]), \\
& \partial_{\Sigma, i+1}^{*}([1] \otimes[F])=\sum_{j \in V \backslash F} \varepsilon(j, \tilde{F} \cup j) \frac{\uparrow^{d-i}\left(x_{\tilde{F} \cup j}\right)}{\uparrow^{d-i+1}\left(x_{\tilde{F} \cup j}\right)}[1] \otimes[F \cup j] \\
& =-\sum_{j \in V \backslash F} \varepsilon(j, F \cup j) \frac{\uparrow^{d-i}\left(x_{1,1}\right)}{\uparrow^{d-i+1}\left(x_{1,1}\right)} \frac{\uparrow^{d-i+1}\left(x_{F \cup j}\right)}{\uparrow^{d-i+2}\left(x_{F}\right)}[1] \otimes[F \cup j] \\
& =-\frac{x_{d-i+1,1}}{x_{d-i+2,1}} \cdot \uparrow\left(\sum_{j \in V \backslash F} \varepsilon(j, F \cup j) \frac{\uparrow^{d-i}\left(x_{F \cup j}\right)}{\uparrow^{d-i+1}\left(x_{F}\right)}[1] \otimes[F \cup j]\right) \\
& =\left(-\frac{x_{d-i+1,1}}{x_{d-i+2,1}} \mathrm{id} \otimes \uparrow \boldsymbol{\partial}_{\Delta, i}^{*}\right)([1] \otimes[F]) .
\end{aligned}
$$

7.2. Eigenvectors of cones. In order to describe the Laplacian eigenvalues and eigenvectors of $1 * \Delta$ in terms of those of $\Delta$, we first need some basic facts about the Laplacians of an arbitrary simplicial complex. The following proposition does not depend on fine weighting and works with any weighted boundary map that satisfies $\partial^{2}=0$. 
Proposition 7.1. Let $\Omega^{d}$ be a simplicial complex, and let $-1 \leq i \leq d$. Let $\mathbf{L}_{i}^{\mathrm{ud}}=$ $\mathbf{L}_{\Omega, i}^{\mathrm{ud}}, \mathbf{L}_{i}^{\mathrm{du}}=\mathbf{L}_{\Omega, i}^{\mathrm{du}}, \boldsymbol{\partial}_{i}=\boldsymbol{\partial}_{\Omega, i}$, and $\boldsymbol{\partial}_{i}^{*}=\boldsymbol{\partial}_{\Omega, i}^{*}$.

(1) The chain group $C_{i}(\Omega)$ decomposes as a direct sum

$$
C_{i}(\Omega)=C_{i}^{\mathrm{ud}}(\Omega) \oplus C_{i}^{\mathrm{du}}(\Omega) \oplus C_{i}^{0}(\Omega),
$$

where

- $C_{i}^{\mathrm{ud}}(\Omega)$ has a basis consisting of eigenvectors of $\mathbf{L}_{i}^{\mathrm{ud}}$ whose eigenvalues are all nonzero, and on which $\mathbf{L}_{i}^{\mathrm{du}}$ acts by zero;

- $C_{i}^{\mathrm{du}}(\Omega)$ has a basis consisting of eigenvectors of $\mathbf{L}_{i}^{\mathrm{du}}$ whose eigenvalues are all nonzero, and on which $\mathbf{L}_{i}^{\mathrm{ud}}$ acts by zero; and

- $\mathbf{L}_{i}^{\mathrm{ud}}$ and $\mathbf{L}_{i}^{\mathrm{du}}$ both act by zero on $C_{i}^{0}(\Omega)$.

(2) $\operatorname{ker}\left(\mathbf{L}_{i}^{\mathrm{ud}}\right)=\operatorname{ker}\left(\boldsymbol{\partial}_{i+1}^{*}\right)$ and $\operatorname{ker}\left(\mathbf{L}_{i}^{\mathrm{du}}\right)=\operatorname{ker}\left(\boldsymbol{\partial}_{i}\right)$.

(3) $\operatorname{dim} C_{i}^{0}(\Omega)=\tilde{\beta}_{i}(\Omega)$, the $i^{\text {th }}$ Betti number of $\Omega$.

(4) Each of the spectra $\mathbf{s}_{i}^{\mathrm{ud}}, \mathbf{s}_{i}^{\mathrm{du}}, \mathbf{s}_{i}^{\text {tot }}$ of $\Omega$ has cardinality $f_{i}(\Omega)$ as a multiset, and

$$
\begin{aligned}
& \mathbf{s}_{i}^{\mathrm{du}} \stackrel{\circ}{=} \mathbf{s}_{i-1}^{\mathrm{ud}}, \quad \text { and } \\
& \mathbf{s}_{i}^{\mathrm{tot}} \stackrel{\circ}{=} \mathbf{s}_{i}^{\mathrm{ud}} \cup \mathbf{s}_{i}^{\mathrm{du}} \stackrel{\circ}{=} \mathbf{s}_{i}^{\mathrm{ud}} \cup \mathbf{s}_{i-1}^{\mathrm{ud}} .
\end{aligned}
$$

Proof. For assertion (1), note that $\mathbf{L}_{i}^{\mathrm{ud}} \mathbf{L}_{i}^{\mathrm{du}}=\boldsymbol{\partial} \boldsymbol{\partial}^{*} \boldsymbol{\partial}^{*} \boldsymbol{\partial}=0$ and $\mathbf{L}_{i}^{\mathrm{du}} \mathbf{L}_{i}^{\mathrm{ud}}=\boldsymbol{\partial}^{*} \boldsymbol{\partial} \boldsymbol{\partial} \boldsymbol{\partial}^{*}$ $=0$. Thus we may simply take $C_{i}^{\mathrm{ud}}(\Omega)$ and $C_{i}^{\mathrm{du}}(\Omega)$ to be the spans of the eigenvectors of $\mathbf{L}_{i}^{\mathrm{ud}}$ and $\mathbf{L}_{i}^{\mathrm{du}}$ with nonzero eigenvalues.

For assertion (2), the operators $\mathbf{L}_{i}^{\text {ud }}$ and $\boldsymbol{\partial}_{i+1}^{*}$ act on the same space, namely $C_{i}(\Omega)$, and they have the same rank (this is just the linear algebra fact that $\operatorname{rank}\left(M M^{T}\right)=\operatorname{rank} M$ for any matrix $\left.M\right)$. Therefore, their kernels have the same dimension. Clearly $\operatorname{ker}\left(\mathbf{L}_{i}^{\mathrm{ud}}\right) \supseteq \operatorname{ker}\left(\boldsymbol{\partial}_{i+1}^{*}\right)$, so we must have equality. The same argument shows that $\operatorname{ker}\left(\mathbf{L}_{i}^{\mathrm{du}}\right)=\operatorname{ker}\left(\boldsymbol{\partial}_{i}\right)$.

Assertion (3) follows from the calculation

$$
\begin{aligned}
\tilde{\beta}_{i}(\Omega)=\operatorname{dim} \tilde{H}_{i}(\Omega, \mathbb{Q}) & =\left(\operatorname{dim} \operatorname{ker} \boldsymbol{\partial}_{i}\right)-\left(\operatorname{dim} \operatorname{im} \boldsymbol{\partial}_{i+1}\right) \\
& =\left(f_{i}-\operatorname{rank} \boldsymbol{\partial}_{i}\right)-\left(\operatorname{rank} \boldsymbol{\partial}_{i+1}\right) \\
& =\operatorname{dim} C_{i}-\operatorname{dim} C_{i}^{\mathrm{ud}}-\operatorname{dim} C_{i}^{\mathrm{du}}=\operatorname{dim} C_{i}^{0} .
\end{aligned}
$$

For assertion (4), first note that $\operatorname{dim} C_{i}(\Omega)=f_{i}(\Omega)$, and that the matrix representing each spectrum is symmetric, hence diagonalizable. The identity (33a) is a standard fact in linear algebra, and (33b) is a consequence of the decomposition (32).

Proposition 7.2. As in Section 7.1, let $\Delta^{d}$ be a simplicial complex on the vertex set $[2, n]$, and let $\Sigma=1 * \Delta$. Then

$$
\begin{aligned}
\mathbf{s}_{i}^{\mathrm{ud}}(\Sigma) \stackrel{\circ}{=} & \left.X_{d-i+1,1}+\uparrow \lambda: \lambda \in \mathbf{s}_{i}^{\mathrm{ud}}(\Delta), \lambda \neq 0\right\} \\
& \cup\left\{X_{d-i+1,1}+\frac{X_{d-i+1,1}}{X_{d-i+2,1}} \uparrow \mu: \mu \in \mathbf{s}_{i-1}^{\mathrm{ud}}(\Delta), \mu \neq 0\right\} \\
& \cup\left\{X_{d-i+1,1}\right\}^{\tilde{\beta}_{i}(\Delta)} .
\end{aligned}
$$

Here the symbol $\cup$ denotes multiset union, and the superscript in the last line indicates multiplicity. 
Proof. Throughout the proof, we abbreviate $\mathbf{L}_{\Sigma, i}^{\mathrm{ud}}$ by $\mathbf{L}$. All other Laplacians that arise will be specified precisely.

First, let $V \in C_{i}^{\text {ud }}(\Delta)$ be an eigenvector of $\mathbf{L}_{\Delta, i}^{\text {ud }}$ with eigenvalue $\lambda \neq 0$. Then $\uparrow \mathbf{L}_{\Delta, i}^{\mathrm{ud}}(\uparrow V)=\uparrow \lambda \uparrow V$, and, by Lemma 6.2

$$
\uparrow \partial_{\Delta, i}(\uparrow V)=\frac{1}{\uparrow \lambda} \uparrow \partial_{\Delta, i}\left(\uparrow \partial_{\Delta, i+1}\left(\uparrow \partial_{\Delta, i+1}^{*}(\uparrow V)\right)\right)=0 .
$$

Using (31a)... (31d) and (35), we calculate

$$
\begin{aligned}
\mathbf{L}([\emptyset] \otimes \uparrow V)= & \boldsymbol{\partial}_{\Sigma, i}\left(\boldsymbol{\partial}_{\Sigma, i}^{*}([\emptyset] \otimes \uparrow V)\right) \\
= & \boldsymbol{\partial}_{\Sigma, i}\left(\uparrow{ }^{d-i} \boldsymbol{\partial}_{\Gamma}^{*}[\emptyset] \otimes \uparrow V+[\emptyset] \otimes \uparrow \boldsymbol{\partial}_{\Delta, i+1}^{*}(\uparrow V)\right) \\
= & x_{d-i+1,1} \boldsymbol{\partial}_{\Sigma, i+1}([1] \otimes \uparrow V)+\boldsymbol{\partial}_{\Sigma, i+1}\left([\emptyset] \otimes \uparrow \boldsymbol{\partial}_{\Delta, i+1}^{*}(\uparrow V)\right) \\
= & x_{d-i+1,1}\left(\uparrow^{d-i} \boldsymbol{\partial}_{\Gamma}[1] \otimes \uparrow V-\frac{x_{d-i+1,1}}{x_{d-i+2,1}}[1] \otimes \uparrow \boldsymbol{\partial}_{\Delta, i}(\uparrow V)\right) \\
& +[\emptyset] \otimes \uparrow \boldsymbol{\partial}_{\Delta, i+1}\left(\uparrow \boldsymbol{\partial}_{\Delta, i+1}^{*}(\uparrow V)\right) \\
= & \left(X_{d-i+1,1}+\uparrow \lambda\right)([\emptyset] \otimes \uparrow V) .
\end{aligned}
$$

Therefore, $[\emptyset] \otimes \uparrow V \in C_{i}(\Sigma)$ is an eigenvector of $\mathbf{L}$, with eigenvalue $X_{d-i+1,1}+\uparrow \lambda$. Notice that this eigenvalue cannot be zero: since $1 \notin \Delta$, no Laplacian eigenvalue of $\Delta$ can possibly equal $-X_{d-i, 1}$.

Second, let $W$ be an eigenvector in $C_{i}^{\mathrm{du}}(\Delta)$ with nonzero eigenvalue $\mu$. That is, $\mathbf{L}_{\Delta_{i}}^{\mathrm{du}}(W)=\boldsymbol{\partial}_{\Delta, i}^{*}\left(\boldsymbol{\partial}_{\Delta, i}(W)\right)=\mu W$, and $\boldsymbol{\partial}_{\Delta, i+1}^{*}(W)=0$ by a computation similar to (35). Define

$$
A=[\emptyset] \otimes \uparrow W, \quad B=[1] \otimes \uparrow \partial_{\Delta, i}(\uparrow W) .
$$

Note that these are both nonzero elements of $C_{i}(\Sigma)$. Then

$$
\begin{aligned}
\mathbf{L}(A) & =\boldsymbol{\partial}_{\Sigma, i+1}\left(\boldsymbol{\partial}_{\Sigma, i+1}^{*}([\emptyset] \otimes \uparrow W)\right) \\
& =\boldsymbol{\partial}_{\Sigma, i+1}\left(\uparrow^{d-i} \boldsymbol{\partial}_{\Gamma}^{*}[\emptyset] \otimes \uparrow W+[\emptyset] \otimes \uparrow \boldsymbol{\partial}_{\Delta, i+1}^{*}(\uparrow W)\right) \\
& =x_{d-i+1,1} \boldsymbol{\partial}_{\Sigma, i+1}([1] \otimes \uparrow W) \\
& =x_{d-i+1,1}\left(\uparrow^{d-i} \boldsymbol{\partial}_{\Gamma}[1] \otimes \uparrow W-\frac{x_{d-i+1,1}}{x_{d-i+2,1}}[1] \otimes \uparrow \boldsymbol{\partial}_{\Delta, i}(\uparrow W)\right) \\
& =X_{d-i+1,1} A-\left(\frac{X_{d-i+1,1}}{x_{d-i+2,1}}\right) B
\end{aligned}
$$

and

$$
\begin{aligned}
\mathbf{L}(B) & =\boldsymbol{\partial}_{\Sigma, i+1}\left(\boldsymbol{\partial}_{\Sigma, i+1}^{*}\left([1] \otimes \uparrow \boldsymbol{\partial}_{\Delta, i}(\uparrow W)\right)\right) \\
& =\boldsymbol{\partial}_{\Sigma, i+1}\left(-\frac{x_{d-i+1,1}}{x_{d-i+2,1}}[1] \otimes \uparrow \boldsymbol{\partial}_{\Delta, i}^{*}\left(\uparrow \boldsymbol{\partial}_{\Delta, i}(\uparrow W)\right)\right) \\
& =-\frac{x_{d-i+1,1}}{x_{d-i+2,1}} \uparrow \cdot \boldsymbol{\partial}_{\Sigma, i+1}([1] \otimes \uparrow W) \\
& =\left(-\frac{X_{d-i+1,1}}{x_{d-i+2,1}} \uparrow \mu\right) A+\left(\frac{X_{d-i+1,1}}{X_{d-i+2,1}} \uparrow \mu\right) B,
\end{aligned}
$$

where the last step follows by the equality of (37a) and (37b). Letting

$$
f=X_{d-i+1,1}, \quad g=-\frac{X_{d-i+1,1}}{x_{d-i+2,1}}, \quad h=-\frac{\uparrow \mu}{x_{d-i+2,1}},
$$


the calculations above say that

$$
\mathbf{L}(A)=f A+g B, \quad \mathbf{L}(B)=h(f A+g B),
$$

which implies that

$\mathbf{L}(f A+g B)=f \mathbf{L}(A)+g \mathbf{L}(B)=f(f A+g B)+g h(f A+g B)=(f+g h)(f A+g B)$.

That is, $f A+g B$ is an eigenvector of $\mathbf{L}$ with eigenvalue

$$
f+g h=X_{d-i+1,1}+\frac{X_{d-i+1,1}}{X_{d-i+2,1}} \uparrow \mu .
$$

As before, this quantity cannot be zero because $\Delta$ does not contain the vertex 1 .

Third, let $Z \in C_{i}^{0}(\Delta)$; we will show that $[\emptyset] \otimes \uparrow Z$ is an eigenvector of $\mathbf{L}$ with eigenvalue $X_{d-i+1,1}$. Indeed, by (2) of Proposition 7.1 we have $\boldsymbol{\partial}_{\Delta, i}(Z)=\boldsymbol{\partial}_{\Delta, i+1}^{*}(Z)=$ 0 , so that

$$
\uparrow \partial_{\Delta, i}(\uparrow Z)=\uparrow \partial_{\Delta, i+1}^{*}(\uparrow Z)=0 .
$$

Therefore

$$
\begin{aligned}
\mathbf{L}([\emptyset] \otimes \uparrow Z) & =\boldsymbol{\partial}_{\Sigma, i+1}\left(\boldsymbol{\partial}_{\Sigma, i+1}^{*}([\emptyset] \otimes \uparrow Z)\right) \\
& =\boldsymbol{\partial}_{\Sigma, i+1}\left(\left(\uparrow{ }^{d-i} \boldsymbol{\partial}_{\Gamma}^{*}[\emptyset] \otimes \uparrow Z\right)+[\emptyset] \otimes \uparrow \boldsymbol{\partial}_{\Delta, i+1}^{*}(\uparrow Z)\right) \\
& =x_{d-i+1,1} \boldsymbol{\partial}_{\Sigma, i+1}([1] \otimes \uparrow Z) \\
& =x_{d-i+1,1} \cdot\left(\uparrow^{d-i} \boldsymbol{\partial}_{\Gamma}[1] \otimes \uparrow Z-\frac{x_{d-i+1,1}}{x_{d-i+2}}[1] \otimes \uparrow \boldsymbol{\partial}_{\Delta, i}(\uparrow Z)\right) \\
& =X_{d-i+1,1}([\emptyset] \otimes \uparrow Z),
\end{aligned}
$$

as desired. By assertion (3) of Proposition 7.1, the multiplicity of this eigenvalue is $\operatorname{dim} C_{i}^{0}(\Delta)=\tilde{\beta}_{i}(\Delta)$.

At this point, we have proven that (34) is true if "으" is replaced with " $\supseteq$ ". On the other hand, we have accounted for

$$
\operatorname{dim} C_{i}^{\mathrm{ud}}(\Delta)+\operatorname{dim} C_{i}^{\mathrm{du}}(\Delta)+\operatorname{dim} C_{i}^{0}(\Delta)=\operatorname{dim} C_{i}(\Delta)=f_{i}(\Delta)
$$

nonzero eigenvalues in $\mathbf{s}_{i}^{\mathrm{ud}}(\Sigma)$. Since the preceding calculations hold for all $i$, we also know $f_{i-1}(\Delta)$ nonzero eigenvalues of $\mathbf{L}_{\Sigma, i-1}^{\mathrm{ud}}\left(\stackrel{\circ}{=} \mathbf{L}_{\Sigma, i}^{\mathrm{du}}\right)$. By (33b), we have accounted for all $f_{i}(\Delta)+f_{i-1}(\Delta)=f_{i}(\Sigma)=\operatorname{dim} C_{i}(\Sigma)$ eigenvalues of $\mathbf{L}_{\Sigma, i}^{\text {tot }}$. So we have indeed found all the nonzero eigenvalues of $\mathbf{L}$.

One can obtain explicit formulas for the spectra $\mathbf{s}_{i}^{\mathrm{du}}(\Sigma)$ and $\mathbf{s}_{i}^{\mathrm{tot}}(\Sigma)$ by applying (33a) and (33b) to the formula (34); we omit the details.

7.3. Eigenvalues of near-cones. The next step is to establish a recurrence (Proposition (7.6) for the Laplacian eigenvalues of near-cones, in terms of the eigenvalues of the deletion and the link of the apex. Our method is based on that of Lemma 5.3 of [13]. By itself, Proposition 7.6 is not a proper recurrence, in the sense that it computes the eigenvalues of a pure complex in terms of complexes that are not necessarily pure. Therefore, it cannot be applied recursively; the proper recurrence for shifted complexes will have to wait until Theorem 8.2 . Since we will be comparing 
complexes with similar face sets but of different dimensions, we begin by describing how their Laplacian spectra are related.

Lemma 7.3. Let $\Sigma^{d}$ be a simplicial complex, and let $j<i \leq d$. Then $\mathbf{s}_{j}^{\mathrm{ud}}(\Sigma)=$ $\uparrow^{d-i} \mathbf{s}_{j}^{\mathrm{ud}}\left(\Sigma_{(i)}\right)$.

Proof. The complexes $\Sigma_{(i)}$ and $\Sigma$ have the same face sets for every dimension $\leq i$, but $\operatorname{dim} \Sigma_{(i)}=\operatorname{dim} \Sigma-(d-i)$. Therefore, the algebraic finely weighted boundary maps and Laplacians of $\Sigma$ can be obtained from those of $\Sigma_{(i)}$ by applying $\uparrow^{d-i}$, from which the lemma follows.

Recall that the pure $i$-skeleton of $\Sigma$ is the subcomplex $\Sigma_{[i]}$ generated by the $i$-dimensional faces of $\Sigma$.

Lemma 7.4. Let $\Sigma^{d}$ be a simplicial complex. Then $\mathbf{s}_{d-1}^{\mathrm{ud}}(\Sigma) \stackrel{\circ}{=} \mathbf{s}_{d-1}^{\mathrm{ud}}\left(\Sigma_{[d]}\right)$.

Proof. This result is proved in [12, Lemma 3.2], but we sketch the proof here for completeness. First, observe that $\mathbf{L}_{d-1}^{\text {ud }}$ depends only on $(d-1)$ - and $d$-dimensional faces. Letting $\Omega=\Sigma_{[d]}$, we have $\Omega_{d}=\Sigma_{d}$ and $\Omega_{d-1} \subseteq \Sigma_{d-1}$, and indeed $\mathbf{L}_{\Sigma, d-1}^{\text {ud }}[F]=\mathbf{L}_{\Omega, d-1}^{\text {ud }}[F]$ for any $F \in \Omega_{d-1}$. On the other hand, $\Sigma_{d-1} \backslash \Omega_{d-1}$ consists precisely of those faces $G$ not contained in any $d$-dimensional faces of $\Sigma$. But $\mathbf{L}_{\Sigma, d-1}^{\text {ud }}$ acts by zero on any such $G$, and the lemma follows immediately.

Corollary 7.5. Let $\Sigma^{d}$ be a simplicial complex. Then $\mathbf{s}_{i-1}^{\mathrm{ud}}(\Sigma) \stackrel{\circ}{=} \uparrow^{d-i} \mathbf{s}_{i-1}^{\mathrm{ud}}\left(\Sigma_{[i]}\right)$.

Proof. We have $\mathbf{s}_{i-1}^{\text {ud }}(\Sigma)=\uparrow^{d-i} \mathbf{s}_{i-1}^{\text {ud }}\left(\Sigma_{(i)}\right) \stackrel{\circ}{=} \uparrow^{d-i} \mathbf{s}_{i-1}^{\text {ud }}\left(\left(\Sigma_{(i)}\right)_{[i]}\right)=\uparrow^{d-i} \mathbf{s}_{i-1}^{\text {ud }}\left(\Sigma_{[i]}\right)$ by Lemmas 7.3 and 7.4. (Note that $\left(\Sigma_{(i)}\right)_{[i]}=\Sigma_{[i]}$.)

Proposition 7.6. Let $\Sigma^{d}$ be a pure near-cone with apex $p$, and let $\Delta=\operatorname{del}_{p} \Sigma$ and $\Lambda=\operatorname{link}_{p} \Sigma$ be the deletion and link, respectively, of $\Sigma$ with respect to vertex $p$. Then

$$
\begin{aligned}
\mathbf{s}_{d-1}^{\mathrm{ud}}(\Sigma) \stackrel{\circ}{=} & \left.X_{1, p}+\lambda: \lambda \in \mathbf{s}_{d-1}^{\mathrm{ud}}(\Delta), \lambda \neq 0\right\} \\
& \cup\left\{X_{1, p}+\frac{X_{1, p}}{X_{2, p}} \uparrow \mu: \mu \in \mathbf{s}_{d-2}^{\mathrm{ud}}(\Lambda), \mu \neq 0\right\} \\
& \cup\left\{X_{1, p}\right\}^{\tilde{\beta}_{d-1}(\Delta)} .
\end{aligned}
$$

Proof. If $\Sigma$ is a cone, then the result follows from a direct application of Proposition [7.2. (Note that $\mathbf{s}_{d-1}^{\text {ud }}(\Delta)$ consists of only 0 's in this case, since $\Delta$ is only $(d-1)$-dimensional.) Thus, we may as well assume for the remainder of the proof that $\Sigma$ is not a cone. In this case, $\operatorname{dim} \Delta=d$ and $\operatorname{dim} \Lambda=d-1$. It is not difficult to see that

$$
\begin{aligned}
\Delta_{(d-1)} & =\Lambda, \text { and } \\
\Sigma_{(d)}=\Sigma & =(p * \Delta)_{(d)} .
\end{aligned}
$$


Applying Lemma 7.3 to equation (40) (keeping in mind that $\operatorname{dim}(p * \Delta)=d+1$ ), and then applying Proposition 7.2, we find that

$$
\begin{aligned}
& \uparrow \mathbf{s}_{d-1}^{\mathrm{ud}}(\Sigma)=\mathbf{s}_{d-1}^{\mathrm{ud}}(p * \Delta) \\
& \stackrel{\circ}{=}\left\{X_{2, p}+\uparrow \lambda: \lambda \in \mathbf{s}_{d-1}^{\mathrm{ud}}(\Delta), \lambda \neq 0\right\} \\
& \cup\left\{X_{2, p}+\frac{X_{2, p}}{X_{3, p}} \uparrow \mu: \mu \in \mathbf{s}_{d-2}^{\mathrm{ud}}(\Delta), \mu \neq 0\right\} \\
& \cup\left\{X_{2, p}\right\}^{\tilde{\beta}_{d-1}(\Delta)}
\end{aligned}
$$

so that

$$
\begin{aligned}
\mathbf{s}_{d-1}^{\mathrm{ud}}(\Sigma)=\{ & \left.X_{1, p}+\lambda: \lambda \in \mathbf{s}_{d-1}^{\mathrm{ud}}(\Delta), \lambda \neq 0\right\} \\
& \cup\left\{X_{1, p}+\frac{X_{1, p}}{X_{2, p}} \mu: \mu \in \mathbf{s}_{d-2}^{\mathrm{ud}}(\Delta), \mu \neq 0\right\} \\
& \cup\left\{X_{1, p}\right\}^{\tilde{\beta}_{d-1}(\Delta)} .
\end{aligned}
$$

The desired result now follows from applying Lemma 7.3 to equation (39).

\section{The Laplacian SPECtrum of a Shifted COMPlex}

In this section, we explicitly describe the eigenvalues of the algebraic finely weighted Laplacians of a shifted complex $\Sigma$. The eigenvalues are Laurent polynomials called $z$-polynomials, which are in one-to-one correspondence with the critical pairs of the complex: pairs $(A, B)$ such that $A \in \Sigma, B \notin \Sigma$, and $B$ covers $A$ in the componentwise partial order. The main result, Theorem 1.5, is proved by establishing identical recurrences for the $z$-polynomials (Theorem [8.2) and critical pairs (Corollary 8.8).

8.1. $z$-polynomials. Let $S$ and $T$ be multisets of integers. Define a Laurent polynomial $z(S, T)$ by the formula

$$
z(S, T)=\frac{1}{\uparrow X_{S}} \sum_{j \in T} X_{S \cup j}
$$

where as usual the symbol $\cup$ denotes multiset union. An example was given at the end of Example 1.7

Proposition 8.1. Let $d>i$ be integers, and let $S, T$ be sets of integers greater than $p$. Then

$$
X_{d-i+1, p}+\uparrow^{d-i} z(S, T)=\uparrow^{d-i} z(S, \tilde{T})
$$

and

$$
X_{d-i+1, p}+\frac{X_{d-i+1, p}}{X_{d-i+2, p}} \uparrow^{d-i+1} z(S, T)=\uparrow^{d-i} z(\tilde{S}, \tilde{T}),
$$

where $\tilde{S}=S \cup\{p\}$ and $\tilde{T}=T \cup\{p\}$. 
Proof. We will use the identity (19a) repeatedly in the calculations. For (42), observe that

$$
\begin{aligned}
& X_{d-i+1, p}+\uparrow^{d-i} z(S, T)=X_{d-i+1, p}+\frac{1}{\uparrow^{d-i+1} X_{S}} \sum_{j \in T} \uparrow^{d-i} X_{S \cup j} \\
& =\frac{1}{\uparrow^{d-i+1} X_{S}}\left(X_{d-i+1, p} \cdot \uparrow^{d-i+1} X_{S}+\sum_{j \in T} \uparrow^{d-i} X_{S \cup j}\right) \\
& =\frac{1}{\uparrow^{d-i+1} X_{S}}\left(\uparrow^{d-i} X_{\tilde{S}}+\sum_{j \in T} \uparrow^{d-i} X_{S \cup j}\right) \\
& =\frac{1}{\uparrow^{d-i+1} X_{S}} \sum_{j \in \tilde{T}} \uparrow^{d-i} X_{S \cup j} \\
& =\uparrow^{d-i} z(S, \tilde{T}),
\end{aligned}
$$

and for (43), observe that

$$
\begin{aligned}
X_{d-i+1, p} & +\frac{X_{d-i+1, p}}{X_{d-i+2, p}} \uparrow^{d-i+1} z(S, T) \\
& =X_{d-i+1, p}+\frac{X_{d-i+1, p}}{X_{d-i+2, p} \cdot \uparrow^{d-i+2} X_{S}} \sum_{j \in T} \uparrow^{d-i+1} X_{S \cup j} \\
& =X_{d-i+1, p}+\frac{X_{d-i+1, p}}{\uparrow^{d-i+1} X_{\tilde{S}}} \sum_{j \in T} \uparrow^{d-i+1} X_{S \cup j} \\
& =\frac{1}{\uparrow^{d-i+1} X_{\tilde{S}}}\left(X_{d-i+1, p} \cdot \uparrow^{d-i+1} X_{\tilde{S}}+\sum_{j \in T} X_{d-i+1, p} \cdot \uparrow^{d-i+1} X_{S \cup j}\right) \\
& =\frac{1}{\uparrow^{d-i+1} X_{\tilde{S}}}\left(\uparrow^{d-i} X_{\tilde{S} \cup p}+\sum_{j \in T} \uparrow^{d-i} X_{\tilde{S} \cup j}\right) \\
& =\frac{1}{\uparrow^{d-i+1} X_{\tilde{S}}} \sum_{j \in \tilde{T}} \uparrow^{d-i} X_{\tilde{S} \cup j} \\
& =\uparrow^{d-i} z(\tilde{S}, \tilde{T}) .
\end{aligned}
$$

Theorem 8.2. Let $\Sigma^{d}$ be a shifted simplicial complex. Then every nonzero eigenvalue of $\mathbf{s}_{i-1}^{\mathrm{ud}}(\Sigma)$ has the form of a z-polynomial. Moreover, the spectrum $\mathbf{s}_{i-1}^{\mathrm{ud}}(\Sigma)$ is determined recursively as follows.

If $\Sigma$ has no vertices, then $\mathbf{s}_{i-1}^{\mathrm{ud}}(\Sigma)$ has no nonzero elements.

Otherwise,

$$
\begin{aligned}
\mathbf{s}_{i-1}^{\mathrm{ud}}(\Sigma) & \stackrel{\circ}{=}\left\{\uparrow^{d-i} z(S, \tilde{T}): 0 \neq z(S, T) \in \mathbf{s}_{i-1}^{\mathrm{ud}}(\Delta)\right\} \\
& \cup\left\{\uparrow^{d-i} z(\tilde{S}, \tilde{T}): 0 \neq z(S, T) \in \mathbf{s}_{i-2}^{\mathrm{ud}}(\Lambda)\right\} \cup\left\{\uparrow^{d-i} z(\emptyset, \tilde{\emptyset})\right\}^{\tilde{\beta}_{i-1}(\Delta)}
\end{aligned}
$$

where $p$ is the initial vertex of $\Sigma_{[i]} ; \tilde{S}=S \cup p ; \tilde{T}=T \cup p ; \Delta=\operatorname{del}_{p} \Sigma_{[i]}$; and $\Lambda=\operatorname{link}_{p} \Sigma_{[i]}$. 
Proof. The proof is by induction on the number of vertices of $\Sigma$. When $\Sigma$ has no vertices, $\Sigma$ is either the empty complex with no faces, or the trivial complex whose only face is the empty face. In either case, $\mathbf{s}_{i-1}^{\text {ud }}(\Sigma)$ has no nonzero elements.

We now assume that $\Sigma$ has at least one vertex. Then

$$
\begin{aligned}
\mathbf{s}_{i-1}^{\mathrm{ud}}\left(\Sigma_{[i]}\right) \stackrel{\circ}{=} & \left.X_{1, p}+\lambda: \lambda \in \mathbf{s}_{i-1}^{\mathrm{ud}}(\Delta), \lambda \neq 0\right\} \\
& \cup\left\{X_{1, p}+\frac{X_{1, p}}{X_{2, p}} \uparrow \mu: \mu \in \mathbf{s}_{i-2}^{\mathrm{ud}}(\Lambda), \mu \neq 0\right\} \\
& \cup\left\{X_{1, p}+0\right\}^{\tilde{\beta}_{i-1}(\Delta)} \\
= & \left\{X_{1, p}+z(S, T): z(S, T) \in \mathbf{s}_{i-1}^{\mathrm{ud}}(\Delta), z(S, T) \neq 0\right\} \\
& \cup\left\{X_{1, p}+\frac{X_{1, p}}{X_{2, p}} z(S, T): z(S, T) \in \mathbf{s}_{i-2}^{\mathrm{ud}}(\Lambda), z(S, T) \neq 0\right\} \\
& \cup\left\{X_{1, p}+z(\emptyset, \emptyset)\right\}^{\tilde{\beta}_{i-1}(\Delta)} \\
= & \left\{z(S, \tilde{T}): 0 \neq z(S, T) \in \mathbf{s}_{i-1}^{\mathrm{ud}}(\Delta)\right\} \\
& \cup\left\{z(\tilde{S}, \tilde{T}): 0 \neq z(S, T) \in \mathbf{s}_{i-2}^{\mathrm{ud}}(\Lambda)\right\} \\
& \cup\{z(\emptyset, \tilde{\emptyset})\}^{\tilde{\beta}_{i-1}(\Delta)} .
\end{aligned}
$$

The $\stackrel{\circ}{=}$-equivalence above is by Proposition 7.6. The following equality is justified by the identity $z(\emptyset, \emptyset)=0$ and induction on the number of vertices, since $\Delta$ and $\Lambda$ each have one fewer vertex than $\Sigma$. Note that $\lambda$ and $\mu$ are each replaced by $z(S, T)$, with no raising operator, because $\operatorname{dim} \Delta \leq i$ and $\operatorname{dim} \Lambda=i-1$. The final equality comes from Proposition 8.1. The result now follows from Corollary 7.5.

8.2. Critical pairs. Throughout this section, let $\mathcal{F}$ be a $k$-family of sets of integers, and let $p$ be the smallest integer occurring in any element of $\mathcal{F}$.

Definition 8.3. A critical pair for $\mathcal{F}$ is an ordered pair $(A, B)$, where $A=\left\{a_{1}<\right.$ $\left.a_{2}<\cdots<a_{k}\right\}$ and $B=\left\{b_{1}<b_{2}<\cdots<b_{k}\right\}$ are sets of integers such that $A \in \mathcal{F}$, $B \notin \mathcal{F}$, and $B$ covers $A$ in componentwise order. That is, $b_{i}=a_{i}+1$ for exactly one $i$, and $b_{j}=a_{j}$ for all $j \neq i$. (Note that $b_{i}$ need not be in the vertex set of $\mathcal{F}$.) The signature of $(A, B)$ is the set of vertices $\sigma(A, B)=\left\{a_{1}, \ldots, a_{i-1}, a_{i}\right\}$. The long signature is the ordered pair of sets $\bar{\sigma}(A, B)=(S, T)$, where $S=\left\{a_{1}, \ldots, a_{i-1}\right\}$ and $T=\left\{j: p \leq j \leq a_{i}\right\}$. The multisets of signatures and long signatures of critical pairs of $\mathcal{F}$ are denoted $\sigma(\mathcal{F})$ and $\bar{\sigma}(\mathcal{F})$ respectively.

As described in the Introduction and Example 1.7, critical pairs are especially significant for shifted simplicial complexes. We will soon see that the critical pairs of a shifted complex are in bijection with the eigenvalues of its algebraic finely weighted Laplacian.

Definition 8.4. The degree of a vertex $v$ in the family $\mathcal{F}$ is $\operatorname{deg}_{\mathcal{F}}(v)=\mid\{F \in$ $\mathcal{F}: v \in F\} \mid$.

Proposition 8.5. Let $\mathcal{F}$ be a shifted family. Then $\operatorname{deg}_{\mathcal{F}}(v)-\operatorname{deg}_{\mathcal{F}}(v+1)$ counts the number of signatures of $\mathcal{F}$ whose greatest element is $v$. 
Proof. Let $S=\{F \in \mathcal{F}: v \in F\}$ and $T=\{F \in \mathcal{F}: v+1 \in F\}$. Partition $S=S_{1} \dot{\cup} S_{2} \dot{\cup} S_{3}$ and $T=T_{1} \dot{\cup} T_{2} \dot{\cup} T_{3}$ as follows:

$$
\begin{aligned}
& S_{1}=\{F \in \mathcal{F}: v, v+1 \in F\}, \\
& S_{2}=\{F \in \mathcal{F}: v \in F, v+1 \notin F, F \backslash\{v\} \dot{\cup}\{v+1\} \in \mathcal{F}\}, \\
& S_{3}=\{F \in \mathcal{F}: v \in F, v+1 \notin F, F \backslash\{v\} \dot{\cup}\{v+1\} \notin \mathcal{F}\}, \\
& T_{1}=\{F \in \mathcal{F}: v, v+1 \in F\}, \\
& T_{2}=\{F \in \mathcal{F}: v \notin F, v+1 \in F, F \backslash\{v+1\} \dot{\cup}\{v\} \in \mathcal{F}\}, \\
& T_{3}=\{F \in \mathcal{F}: v \notin F, v+1 \in F, F \backslash\{v+1\} \dot{\cup}\{v\} \notin \mathcal{F}\} .
\end{aligned}
$$

Then $S_{1}=T_{1}$, and there is an obvious bijection between $S_{2}$ and $T_{2}$. Since $\mathcal{F}$ is shifted, $T_{3}=\emptyset$, so $\operatorname{deg}_{\mathcal{F}}(v)-\operatorname{deg}_{\mathcal{F}}(v+1)=|S|-|T|=\left|S_{3}\right|$.

Finally, if $F \in S_{3}$, then $(F, G)$ is a critical pair, where $G=F \backslash\{v\} \dot{\cup}\{v+1\}$. For each such critical pair, the greatest element of the signature is $v$. Conversely, if $(A, B)$ is a critical pair whose signature's greatest element is $v$, then $A \in S_{3}$.

Corollary 8.6. Let $\Sigma^{d}$ be a pure shifted simplicial complex with initial vertex $p$. Then $\operatorname{deg}_{\Sigma_{d}}(p)-\operatorname{deg}_{\Sigma_{d}}(p+1)=\tilde{\beta}_{d-1}\left(\operatorname{del}_{p} \Sigma\right)$.

Proof. Just as in the proof of Proposition 8.5 above,

$$
\operatorname{deg}_{\Sigma_{d}}(p)-\operatorname{deg}_{\Sigma_{d}}(p+1)=\left|S_{3}\right|,
$$

where

$$
S_{3}=\left\{F \in \Sigma_{d}: p \in F, p+1 \notin F, F \backslash\{p\} \dot{\cup}\{p+1\} \notin \Sigma_{d}\right\} .
$$

There is a bijection between $S_{3}$ and the set

$$
S_{3}^{\prime}=\left\{G \in\left(\operatorname{del}_{p} \Sigma\right)_{d-1}: p+1 \notin G, G \dot{\cup}\{p+1\} \notin \operatorname{del}_{p} \Sigma\right\}
$$

given by $G=F \backslash\{p\}$. Then, by equation (16),

$$
\tilde{\beta}_{d-1}\left(\operatorname{del}_{p} \Sigma\right)=\left|S_{3}^{\prime}\right| \text {. }
$$

Combining the four displayed equations yields the desired result.

Proposition 8.7. Let $\Sigma$ be a shifted complex with initial vertex $p$. Let $\Delta=\operatorname{del}_{p} \Sigma$ and $\Lambda=\operatorname{link}_{p} \Sigma$. Then

$$
\sigma\left(\Sigma_{i}\right)=\sigma\left(\Delta_{i}\right) \cup\left\{p \cup \dot{\cup} F: F \in \sigma\left(\Lambda_{i-1}\right)\right\} \cup\{p\}^{\operatorname{deg}_{\Sigma_{i}}(p)-\operatorname{deg}_{\Sigma_{i}}(p+1)},
$$

where $\cup$ denotes multiset union.

Proof. The multiplicity of the signature $\{p\}$ follows from Proposition 8.5

Suppose that $A \in \Delta_{i}, B \notin \Delta_{i}$, and $B$ covers $A$ in the componentwise partial order. Then $A \in \Sigma_{i}$ and $p \notin A$. Since $p \notin A$ and $A \prec B$, we conclude that $p \notin B$, and so $B \notin \Sigma_{i}$. Hence we have a one-to-one map of multisets

$$
\sigma\left(\Delta_{i}\right) \rightarrow \sigma\left(\Sigma_{i}\right)
$$

On the other hand, suppose that $A \in \Lambda_{i-1}, B \notin \Lambda_{i-1}$, and $B$ covers $A$ in the componentwise partial order. Then $p \notin A$ and $\tilde{A}=A \cup \dot{\cup}\{p\} \in \Sigma_{i}$. The critical pair $(A, B)$ of $\Lambda_{i-1}$ gives rise to the critical pair $(\tilde{A}, \tilde{B})$ of $\Sigma_{i}$, and it is clear that $\sigma(\tilde{A}, \tilde{B})=\sigma(A, B) \dot{\cup}\{p\}$. Furthermore, $\tilde{B} \notin \Sigma_{i}$ because $B \notin \Lambda_{i-1}$. Hence we have a one-to-one map of multisets

$$
\left\{p \dot{\cup} F: F \in \sigma\left(\Lambda_{i-1}\right)\right\} \rightarrow \sigma\left(\Sigma_{i}\right) .
$$


Now we must show, conversely, that every signature $F \neq\{p\}$ of $\Sigma_{i}$ arises in one of these two ways. Let $F$ be such a signature of $\Sigma_{i}$, with critical pair $(A, B)$. So $A \in \Sigma_{i} ; B \notin \Sigma_{i}$ (so $B \notin \Delta_{i}$ ); and $B$ covers $A$ in the componentwise partial order.

First, if $p \notin F$, then $p \notin A$ and so $A \in \Delta_{i}$. Thus $(A, B)$ is a critical pair for $\Delta_{i}$.

Second, if $p \in F$, then $F=F^{\prime} \cup\{p\}$ for some $F^{\prime} \neq \emptyset$. In this case, we have $p \in A$ and $p \in B$ for the critical pair $(A, B)$ whose signature is $F$. Indeed, $p \in F$ directly implies that $p \in A$. If $p \notin B$, then $F=\sigma(A, B)=\{p\}$. Accordingly, let $A^{\prime}=A \backslash\{p\}$ and $B^{\prime}=B \backslash\{p\}$. Then $A^{\prime} \in \Lambda_{i-1}$ and $B \notin \Sigma_{i}$, so $B^{\prime} \notin \Lambda_{i-1}$, and $(A, B)$ is a critical pair for $\Lambda_{i-1}$.

Corollary 8.8. Let $\Sigma^{d}$ be a shifted simplicial complex, and let $i \leq d$. Then the multiset $\bar{\sigma}\left(\Sigma_{i}\right)$ is determined by the following recurrence.

If $\Sigma$ has no vertices, then $\bar{\sigma}\left(\Sigma_{i}\right)=\emptyset$.

Otherwise,

$$
\begin{aligned}
\bar{\sigma}\left(\Sigma_{i}\right)=\{ & \left.(S, \tilde{T}):(S, T) \in \bar{\sigma}\left(\Delta_{i}\right)\right\} \\
& \cup\left\{(\tilde{S}, \tilde{T}):(S, T) \in \bar{\sigma}\left(\Lambda_{i-1}\right)\right\} \\
& \cup\{(\emptyset, \tilde{\emptyset})\}^{\tilde{\beta}_{i-1}(\Delta)}
\end{aligned}
$$

where $p$ is the initial vertex of $\Sigma_{[i]} ; \tilde{S}=S \cup p ; \tilde{T}=T \cup p ; \Delta=\operatorname{del}_{p} \Sigma_{[i]}$; and $\Lambda=\operatorname{link}_{p} \Sigma_{[i]}$.

Proof. If $\sigma(A, B)=\{p\}$, then $\bar{\sigma}(A, B)=(\emptyset,\{p\})=(\emptyset, \tilde{\emptyset})$. So the third term in (45) arises from applying Corollary 8.6 to the pure $i$-dimensional shifted complex $\Sigma_{[i]}$. (Indeed, $\Sigma_{[i]}$ is shifted when $\Sigma$ is, or even just when $\Sigma_{i}$ is.)

For the first two terms in (45), the only hard part is to note that if the first vertex of $\Sigma_{i}$ is $p$, then $p+1$ is the first vertex of $\Delta_{i}$ and $\Lambda_{i-1}$ (unless $\Delta_{i}=\emptyset$, but in that case we don't have to worry about the first set). This accounts for the $\tilde{T}$ 's.

Even though Proposition 8.7 above defines $\Delta$ and $\Lambda$ somewhat differently than here, it is not a problem because $\left(\operatorname{del}_{p} \Sigma\right)_{i}=\left(\operatorname{del}_{p} \Sigma_{[i]}\right)_{i}$ and $\left(\operatorname{link}_{p} \Sigma\right)_{i-1}=$ $\left(\operatorname{link}_{p} \Sigma_{[i]}\right)_{i-1}$. Then, since $\Delta$ and $\Lambda$ only appear as $\Delta_{i}$ and $\Lambda_{i-1}$, it doesn't matter whether we set them to be the deletion and link of $\Sigma$ or of $\Sigma_{[i]}$.

8.3. Theorem 1.5 and its consequences. We can finally prove Theorem 1.5 which characterizes the Laplacian spectra $\mathbf{s}_{i-1}^{\text {ud }}(\Sigma)$ in terms of $z$-polynomials of critical pairs. In the notation we have developed, the theorem can be restated as follows:

Theorem 1.5. Let $\Sigma^{d}$ be a shifted simplicial complex. Then, for each $0 \leq i \leq d$,

$$
\mathbf{s}_{i-1}^{\mathrm{ud}}(\Sigma) \stackrel{\circ}{=} \quad \uparrow^{d-i}\left\{z(S, T):(S, T) \in \bar{\sigma}\left(\Sigma_{i}\right)\right\} .
$$

Proof. Simply note that the recursions in Theorem 8.2 and Corollary 8.8 are identical.

One corollary to Theorem [1.5 is that you can "hear the shape" of a shifted complex (Corollary 8.9), but only if your ears are fine enough (Remark 8.11).

Corollary 8.9. A shifted complex $\Sigma^{d}$ is completely determined by its spectra $\left\{\mathbf{s}_{i-1}^{\text {ud }}(\Sigma)\right\}_{i=0}^{d}$. 
Proof. By Theorem 1.5, the spectra $\left\{\mathbf{s}_{i-1}^{\mathrm{ud}}(\Sigma)\right\}_{i=0}^{d}$ determine the $z$-polynomials $z(S, T)$, and it is easy to see that the long signature $(S, T)$ can be recovered from $z(S, T)$. Furthermore the (short) signature is even more easily recovered from the long signature. When $F=\left\{v_{1}<\ldots<v_{i}\right\}$ is a face of $\Sigma$ that is $\preceq$-maximal, then $\left(F, F \backslash\left\{v_{i}\right\} \cup \dot{\cup}\left\{v_{i+1}\right\}\right)$ is a critical pair, with (short) signature $F$. Thus, among all the (short) signatures of $\Sigma$, we will find all $\preceq$-maximal faces. Furthermore, every (short) signature is a face of $\Sigma$, by definition. Thus, the union $\bigcup_{F \in \sigma(\Sigma)}\{G: G \preceq F\}$ of all $\preceq$-order ideals will yield all the nonempty faces of $\Sigma$.

In fact, if $\Sigma^{d}$ is a pure shifted complex, then it is determined uniquely by its top Laplacian spectrum $\mathbf{s}_{d-1}^{\text {ud }}(\Sigma)$.

Specializing (or "coarsening") the algebraic fine weighting, we obtain, as another corollary to Theorem 1.5. Duval and Reiner's description of the unweighted Laplacian eigenvalues of a shifted complex [13, Thm. 1.1], as we now explain.

The coarse weighting is obtained from the algebraic fine weighting by omitting all first subscripts, i.e., replacing $x_{i, j}=x_{j}$ and $X_{i, j}=X_{j}$. (Thus the monomial corresponding to a facet or set of facets records the degree of each vertex, but forgets the information about the order of the vertices in facets.) Note that if $T=[1, t]$, then every $z$-polynomial $z(S, T)$ specializes to the linear form $E_{t}=X_{1}+\cdots+X_{t}$ in the coarse weighting.

For a partition $\lambda$ (a weakly decreasing list of positive integers), let $E_{\lambda}$ be the multiset in which each part $i$ of $\lambda$ is replaced by $E_{i}$. Recall that the conjugate of $\lambda$ is the partition $\lambda^{\prime}$ in which each part $t$ occurs with multiplicity $\lambda_{t}-\lambda_{t+1}$.

Corollary 8.10. Let $\Sigma^{d}$ be a shifted simplicial complex on the vertices $[n]$. Then

$$
\hat{s}_{d-1}^{\mathrm{ud}}(\Sigma) \stackrel{\circ}{=} E_{\left(\operatorname{deg}_{\Sigma_{d}}\right)^{\prime}},
$$

where the left-hand side denotes the multiset of coarsely weighted Laplacian eigenvalues, and the right-hand side is the conjugate of the partition $\left(\operatorname{deg}_{\Sigma_{d}}(1), \ldots\right.$, $\left.\operatorname{deg}_{\Sigma_{d}}(n)\right)$.

Proof. Theorem 1.5 and the preceding discussion imply that $\hat{s}_{d-1}^{\mathrm{ud}}(\Sigma)$ is $\stackrel{\circ}{=}$-equivalent to the multiset in which $E_{t}$ occurs with multiplicity equal to the number of critical pairs $(A, B)$ of $\Sigma_{d}$ such that $t=\max \sigma(A, B)$. By Proposition 8.5 that multiplicity is $\operatorname{deg}_{\Sigma_{d}}(t)-\operatorname{deg}_{\Sigma_{d}}(t+1)$. The result now follows from the definition of a conjugate partition.

Passing to the unweighted setting by setting $x_{i}=1$ for all $i$ recovers the theorem of Duval and Reiner [13, Thm. 1.1], which states that the Laplacian eigenvalues of a shifted complex $\Sigma$ are given by the conjugate of the partition $\operatorname{deg}_{\Sigma_{d}}$.

Remark 8.11. Duval and Reiner also showed [13, Example 10.2] that there are two nonisomorphic 2-dimensional shifted complexes with the same degree sequence. Corollary 8.10 then shows that, in contrast to Corollary 8.9, the coarsely-weighted eigenvalues are not enough to determine a shifted complex.

8.4. An example: the equatorial bipyramid. To illustrate Theorems 8.2 and 1.5. we calculate the top-dimensional up-down Laplacian spectrum of the bipyramid $B=\langle 235\rangle$ (see Examples 1.7, 3.4 and 5.2). In our recursive calculation, we will 


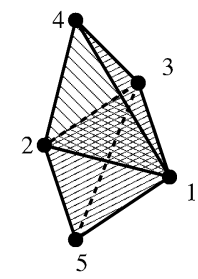

$B_{1}$

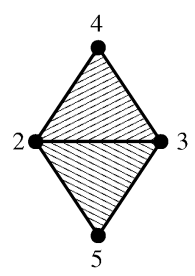

$B_{2}$

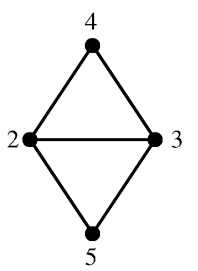

$B_{3}$

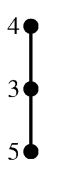

$B_{4}$

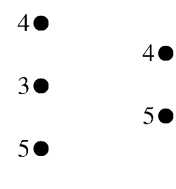

$B_{5} \quad B_{6} \quad B_{7}$

FiguRE 2

TABLE 1

\begin{tabular}{cccccc} 
Subcomplex & Dimension & Vertices & $\begin{array}{c}\text { Critical } \\
\text { pairs }\end{array}$ & Signatures & Eigenvalues \\
\hline$B_{7}$ & 0 & $\{5\}$ & $(5,6)$ & 5 & $z(\emptyset, 5)$ \\
\hline$B_{6}$ & 0 & $\{4,5\}$ & $(5,6)$ & 5 & $z(\emptyset, 45)$ \\
\hline$B_{5}$ & 0 & $\{3,4,5\}$ & $(5,6)$ & 5 & $z(\emptyset, 345)$ \\
\hline$B_{4}$ & 1 & $\{3,4,5\}$ & $(35,45)$ & 3 & $z(\emptyset, 3)$ \\
& 1 & $\{2,3,4,5\}$ & $(25,26)$ & 25 & $z(2,2345)$ \\
\hline$B_{3}$ & & $(35,36)$ & 35 & $z(3,2345)$ \\
& & & $(35,45)$ & 3 & $z(\emptyset, 23)$ \\
\hline$B_{2}$ & 2 & $\{2,3,4,5\}$ & $(235,236)$ & 235 & $z(23,2345)$ \\
& & & $(235,245)$ & 23 & $z(2,23)$ \\
\hline$B_{1}$ & 2 & $\{1,2,3,4,5\}$ & $(125,126)$ & 125 & $z(12,12345)$ \\
& & & $(135,136)$ & 135 & $z(13,12345)$ \\
& & & $(135,145)$ & 13 & $z(1,123)$ \\
& & & $(235,236)$ & 235 & $z(23,12345)$ \\
& & & & 23 & $z(2,123)$ \\
\hline
\end{tabular}

encounter the subcomplexes $B_{2}, \ldots, B_{7}$ of $B=B_{1}$ shown in Figure 2 , and $B_{8}$, the simplicial complex whose only face is the empty face. Observe that

- $B_{1}$ is a near-cone with apex 1 , $\operatorname{del}_{1} B_{1}=B_{2}$, and $\operatorname{link}_{1} B_{1}=B_{3}$;

- $B_{2}=2 * B_{4}$;

- $B_{3}$ is a near-cone with apex $2, \operatorname{del}_{2} B_{3}=B_{4}$, and $\operatorname{link}_{2} B_{3}=B_{5}$;

- $B_{4}=3 * B_{6}$;

- $B_{5}$ is a near-cone with apex $3, \operatorname{del}_{3} B_{5}=B_{6}$, and $\operatorname{link}_{3} B_{5}=B_{8}$;

- $B_{6}$ is a near-cone with apex $4, \operatorname{del}_{4} B_{6}=B_{7}$, and $\operatorname{link}_{4} B_{6}=B_{8}$; and

- $B_{7}=5 * B_{8}$.

The critical pairs, signatures, and nonzero top-dimensional Laplacian eigenvalues of the bipyramid $B=B_{1}$ and its subcomplexes $B_{2}, \ldots, B_{7}$ are listed in Table 1 . This information can be obtained either recursively (using Theorem 8.2 repeatedly) or bijectively (from Theorem 1.5).

We also see from Table 1 that the coarsely-weighted eigenvalues $\hat{s}_{1}^{\text {ud }}\left(B_{1}\right)$ are $\stackrel{\circ}{=}$-equivalent to $E_{5}, E_{5}, E_{5}, E_{3}, E_{3}$ (each $E_{5}$ coming from a $z(S, 12345)$ in $B_{1}$, and 
each $E_{3}$ coming from a $z(S, 123)$ in $\left.B_{1}\right)$, corresponding to the transpose of the degreee sequence of the facets, 55533. (In this case, both the degree sequence and its transpose are 55533.)

\section{Enumerating SPANNing TREeS OF SHIFTED COMPLEXeS}

We now translate Theorem 1.5 from the algebraic to the combinatorial fine weighting, in order to obtain a factorization of the finely weighted spanning tree enumerator $\hat{\tau}(\Sigma)$ of a shifted complex $\Sigma$.

Recall that the long signature $\bar{\sigma}(\mathcal{F})$ of a family $\mathcal{F}$ is the multiset of long signatures of its critical pairs.

Theorem 1.6. Let $\Sigma^{d}$ be a shifted complex with initial vertex $p$. Then:

$$
\begin{aligned}
\hat{\tau}_{d}(\Sigma) & =\left(\prod_{F \in \Lambda_{d-1}} X_{\tilde{F}}\right)\left(\prod_{(S, T) \in \bar{\sigma}\left(\Delta_{d}\right)} \frac{z(S, \tilde{T})}{X_{1, p}}\right) \\
& =\left(\prod_{F \in \Lambda_{d-1}} X_{\tilde{F}}\right)\left(\prod_{(S, T) \in \bar{\sigma}\left(\Delta_{d}\right)} \frac{\sum_{j \in \tilde{T}} X_{S \cup j}}{X_{\tilde{S}}}\right),
\end{aligned}
$$

where $\tilde{F}=F \cup p, \Delta=\operatorname{del}_{p} \Sigma$, and $\Lambda=\operatorname{link}_{p} \Sigma$.

Proof. Since the complex $\Sigma$ is APC, its spanning trees are precisely those of its pure $d$-skeleton $\Sigma_{[d]}$. Similarly, passing from $\Sigma$ to $\Sigma_{[d]}$ does not affect $\Delta_{d}$ or $\Lambda_{d-1}$. Therefore, we may assume without loss of generality that $\Sigma$ is pure.

By Lemma 6.3 and Theorem 1.5. we have

$$
\hat{\tau}_{d}(\Sigma)=\left(\prod_{F \in \operatorname{link}_{p} \Sigma} \uparrow X_{F}\right)\left(\prod_{(S, T) \in \bar{\sigma}\left(\Delta_{d}\right)}\left(X_{1, p}+z(S, T)\right)\right) X_{1, p}^{m},
$$

where $m$ is the number of zero eigenvalues of $\mathbf{L}_{\Delta, d-1}^{\mathrm{ud}}$. Since $\mathbf{L}_{\Delta, d-1}^{\mathrm{ud}}$ acts on $\Delta_{d-1}$, but has $\bar{\sigma}\left(\Delta_{d}\right)$ nonzero eigenvalues (including multiplicity), $m=\left|\Delta_{d-1}\right|-\left|\bar{\sigma}\left(\Delta_{d}\right)\right|=$ $\left|\Lambda_{d-1}\right|-\left|\bar{\sigma}\left(\Delta_{d-1}\right)\right|$, since $\Delta_{d-1}=\Lambda_{d-1}$ by equation (39). Thus,

$$
\begin{aligned}
\hat{\tau}_{d}(\Sigma) & =X_{1, p}^{\left|\Lambda_{d-1}\right|}\left(\prod_{F \in \Lambda_{d-1}}\left(\uparrow X_{F}\right)\right) X_{1, p}^{-\left|\bar{\sigma}\left(\Delta_{d}\right)\right|}\left(\prod_{(S, T) \in \bar{\sigma}\left(\Delta_{d}\right)} X_{1, p}+z(S, T)\right) \\
& =\prod_{F \in \Lambda_{d-1}}\left(X_{1, p} \uparrow X_{F}\right) \prod_{(S, T) \in \bar{\sigma}\left(\Delta_{d}\right)} \frac{X_{1, p}+z(S, T)}{X_{1, p}} .
\end{aligned}
$$

Equation (47) now follows from equations (19a) and (42). Equation (48) then follows from the definition of a $z$-polynomial (41) and from (19a) again, because

$$
\frac{z(S, \tilde{T})}{X_{1, p}}=\frac{1}{X_{1, p}} \frac{\sum_{j \in \tilde{T}} X_{S \cup j}}{\uparrow X_{S}}=\frac{\sum_{j \in \tilde{T}} X_{S \cup j}}{X_{\tilde{S}}} .
$$

Example 9.1. We return to our running example, the equatorial bipyramid $B$. Here $d=2$ and $p=1$, and in the notation of Section 8.4, we have $\Delta=B_{2}$ and 
$\Lambda=B_{3}$. Moreover, $\bar{\sigma}\left(\Delta_{d}\right)=\{(2,23),(23,2345)\}$. Hence equation (48) yields

$$
\begin{aligned}
\hat{\tau}(B)= & X_{123} X_{124} X_{134} X_{125} X_{135}\left(\frac{X_{12}+X_{22}+X_{23}}{X_{12}}\right) \\
& \times\left(\frac{X_{123}+X_{223}+X_{233}+X_{234}+X_{235}}{X_{123}}\right) .
\end{aligned}
$$

Note that this is a genuine polynomial (not just a Laurent polynomial) in the indeterminates $X_{i, j}$.

Corollary 9.2. Let $\Sigma^{d}$ be a shifted complex with initial vertex 1 . Let $\Delta=\operatorname{del}_{1} \Sigma$, $\tilde{\Delta}=1 * \Delta, \Lambda=\operatorname{link}_{1} \Sigma$, and $\tilde{\Lambda}=1 * \Lambda$. Then, in the coarse weighting,

$$
\hat{\tau}_{d}(\Sigma)=X^{\operatorname{deg} \tilde{\Lambda}_{d}} \prod_{i}\left(E_{i} / X_{1}\right)^{\left(\operatorname{deg} \tilde{\Delta}_{d+1}\right)_{i}^{\prime}}
$$

where $E_{i}=X_{1}+\cdots+X_{i}$ and, for a partition $\lambda$, we set $X^{\lambda}:=\prod_{i} X_{i}^{\lambda_{i}}$.

Proof. Upon coarsening the weighting, the first product in (48) becomes $X^{\operatorname{deg} \tilde{\Lambda}_{d}}$ and the second product becomes

$$
\prod_{(S, T) \in \bar{\sigma}\left(\Delta_{d}\right)} \frac{\sum_{j \in \tilde{T}} X_{j}}{X_{1}}=\prod_{(S, T) \in \bar{\sigma}\left(\Delta_{d}\right)} E_{|T|+1} / X_{1} .
$$

We now claim that

$$
\prod_{(S, T) \in \bar{\sigma}\left(\Delta_{d}\right)} E_{|T|+1} / X_{1}=\prod_{(S, T) \in \bar{\sigma}\left(\tilde{\Delta}_{d+1}\right)} E_{|T|} / X_{1} .
$$

Indeed, by Proposition 8.7

$$
\sigma\left(\tilde{\Delta}_{d+1}\right)=\left\{1 \dot{\cup} F: F \in \sigma\left(\Delta_{d}\right)\right\} \cup\{1\}^{m}
$$

for some $m$, since $\Delta=\operatorname{link}_{1}(\tilde{\Delta})$ and $\operatorname{dim}\left(\operatorname{del}_{1}(\tilde{\Delta})\right)=\operatorname{dim} \Delta<d+1$. It now follows that

$$
\prod_{(S, T) \in \bar{\sigma}\left(\tilde{\Delta}_{d+1}\right)} E_{|T|} / X_{1}=\prod_{(S, T) \in \bar{\sigma}\left(\Delta_{d}\right)}\left(E_{|T|+1} / X_{1}\right)\left(X_{1} / X_{1}\right)^{m}
$$

implying the claim. Finally, by Proposition 8.5 and the definition of conjugate partition, we obtain

$\prod_{(S, T) \in \bar{\sigma}\left(\tilde{\Delta}_{d+1}\right)} E_{|T|} / X_{1}=\prod_{t}\left(E_{t} / X_{1}\right)^{\operatorname{leg}_{\tilde{\Delta}_{d+1}}(t)-\operatorname{deg}_{\tilde{\Delta}_{d+1}}(t+1)}=\prod_{i}\left(E_{i} / X_{1}\right)^{\left(\operatorname{deg} \tilde{\Delta}_{d+1}\right)_{i}^{\prime}}$.

Example 9.3. Once again, let $B$ be the equatorial bipyramid. Here $\tilde{\Lambda}_{d}=\{123,124$, $125,134,135\}$. Its degree sequence is 53322 . Thus the monomial factor in Corollary 9.2 is

$$
X_{123} X_{124} X_{125} X_{134} X_{135}=X_{1}^{5} X_{2}^{3} X_{3}^{3} X_{4}^{2} X_{5}^{2} .
$$

Meanwhile, $\tilde{\Delta}_{d+1}=\{1234,1235\}$. Its degree sequence is 22211 , with conjugate 53 . The product factor in Corollary 9.2 is therefore

$$
\left(E_{5} / X_{1}\right)\left(E_{3} / X_{1}\right)=\left(X_{1}+X_{2}+X_{3}+X_{4}+X_{5}\right)\left(X_{1}+X_{2}+X_{3}\right) / X_{1}^{2} .
$$

Putting these terms together yields

$$
\hat{\tau}_{d}(\Sigma)=X_{1}^{3} X_{2}^{3} X_{3}^{3} X_{4}^{2} X_{5}^{2}\left(X_{1}+X_{2}+X_{3}+X_{4}+X_{5}\right)\left(X_{1}+X_{2}+X_{3}\right),
$$

which matches Example 5.2 


\section{Corollaries}

We conclude by showing how several known tree enumerators - for skeletons of simplices, threshold graphs, and Ferrers graphs - can be recovered from our results.

10.1. Skeletons of simplices. Let $\Sigma$ be the $d$-skeleton of the simplex on the vertices $[n]$, so that the set of facets of $\Sigma$ is $\left(\begin{array}{c}{[n]} \\ d+1\end{array}\right)$, the set of all subsets of $[n]$ of cardinality $d+1$. Note that $\Sigma$ is generated as a shifted complex by the single facet $[n-d, n]$. The critical pairs of $\Sigma$ are

$$
\left\{(A \cup\{n\}, A \cup\{n+1\}): A \in\left(\begin{array}{c}
{[n-1]} \\
d
\end{array}\right)\right\}
$$

and the corresponding long signatures are

$$
\bar{\sigma}\left(\Sigma_{d}\right)=\left\{(A,[n]): A \in\left(\begin{array}{c}
{[n-1]} \\
d
\end{array}\right)\right\} .
$$

Setting $\Lambda=\operatorname{link}_{1} \Sigma$ and $\Delta=\operatorname{del}_{1} \Sigma$, we have

$$
\begin{aligned}
\Lambda_{d-1} & =\left(\begin{array}{c}
{[2, n]} \\
d
\end{array}\right) ; \\
\Delta_{d} & =\left(\begin{array}{c}
{[2, n]} \\
d+1
\end{array}\right) ; \\
\bar{\sigma}\left(\Delta_{d}\right) & =\left\{(B,[2, n]): B \in\left(\begin{array}{c}
{[2, n-1]} \\
d
\end{array}\right)\right\} .
\end{aligned}
$$

Applying equation (48), we obtain

$$
\hat{\tau}_{d}(\Sigma)=\left(\prod_{\substack{C \subseteq[2, n] \\|\bar{C}|=d}} X_{\tilde{C}}\right)\left(\prod_{\substack{B \subseteq[2, n-1] \\|B|=d}} \frac{\sum_{j=1}^{n} X_{B \cup j}}{X_{\tilde{B}}}\right) .
$$

The denominators in the second product cancel the factors $X_{\tilde{C}}$ in the first product with $n \notin \tilde{C}$, leaving only those for which $n \in \tilde{C}$. Therefore,

$$
\begin{aligned}
\hat{\tau}_{d}(\Sigma) & =\left(\prod_{\substack{C \subseteq[2, n] \\
n \in C \\
|C|=d}} X_{\tilde{C}}\right)\left(\prod_{\substack{B \subseteq[2, n-1] \\
|B|=d}} \sum_{j=1}^{n} X_{B \cup j}\right) \\
& =\left(\prod_{\substack{C \subseteq[n] \\
1, n \in C \\
|C|=d+1}} X_{C}\right)\left(\prod_{\substack{B \subseteq[2, n-1] \\
|B|=d}} z(B,[n])\right.
\end{aligned}
$$

Passing to the coarse weighting by setting $X_{i, j}=X_{j}$ for every $i, j$, we obtain

$$
\begin{aligned}
\hat{\tau}_{d}(\Sigma) & =\left(\left(X_{1} X_{n}\right)^{\left(\begin{array}{c}
n-2 \\
d-1
\end{array}\right)}\left(X_{2} \cdots X_{n-1}\right)^{\left(\begin{array}{c}
n-3 \\
d-2
\end{array}\right)}\right)\left(\left(X_{2} \cdots X_{n-1}\right)^{\left(\begin{array}{c}
n-3 \\
d-1
\end{array}\right)}\left(X_{1}+\cdots+X_{n}\right)^{\left(\begin{array}{c}
n-2 \\
d
\end{array}\right)}\right) \\
& =\left(X_{1} \cdots X_{n}\right)^{\left(\begin{array}{c}
n-2 \\
d-1
\end{array}\right)}\left(X_{1}+\cdots+X_{n}\right)^{\left(\begin{array}{c}
n-2 \\
d
\end{array}\right)},
\end{aligned}
$$


which is Theorem $3^{\prime}$ of Kalai's paper [20]. Furthermore, setting $X_{i}=1$ for all $i$ recovers Kalai's generalization of Cayley's formula: $\tau_{d}(\Sigma)=n\left(\begin{array}{c}n-2 \\ d\end{array}\right)$.

10.2. Threshold graphs. A threshold graph is a one-dimensional shifted complex $\Sigma$. For simplicity, we assume that the vertex set of $\Sigma$ is $[1, n]$. We may also assume that $\Sigma$ is connected, so that every vertex is adjacent to vertex 1 . Martin and Reiner [27, Theorem 4] found a factorization of the combinatorially finely weighted spanning tree enumerator of $\Sigma$, which may be stated in our notation 5 as:

$$
\hat{\tau}(\Sigma)=X_{\{1, n\}} \prod_{v=2}^{n-1} \sum_{j=1}^{(\operatorname{deg} \Sigma)_{v}^{\prime}} X_{\{v, j\}} .
$$

A somewhat more general result was obtained independently by Remmel and Williamson [31, Theorem 2.4]. We will show how this formula can be recovered from Theorem 1.6 .

The first product in equation (48) is just $X_{\{1,2\}} X_{\{1,3\}} \cdots X_{\{1, n\}}$. For the second product, we must identify the critical pairs of $\Delta=\operatorname{del}_{1} \Sigma$. Note that $\Delta$ is a threshold graph with vertices $[2, n]$.

As is often the case with threshold graphs (and their degree sequences, which we will soon encounter), we need to sort vertices by their relation to the size of the Durfee square of $\Sigma$, the largest square that fits in the Ferrers diagram of its degree sequence. The side length $m$ of the Durfee square is the largest number such that $\{m, m+1\}$ is an edge of $\Sigma$. If $m=1$, then $\Sigma$ is a star graph, the Ferrers diagram of its degree sequence is a hook, and equations (48) and (49) both easily reduce to

$$
\hat{\tau}_{d}(\Sigma)=\prod_{v=2}^{n} X_{\{1, v\}} .
$$

Therefore, we henceforth assume that $m \geq 2$. Note that every edge has at least one endpoint $\leq m$ (because $\{m+1, m+2\} \notin \Sigma$, and that edge is the unique $\preceq$-minimal edge with both endpoints $>m$ ).

For each vertex $v$ of $\Sigma$, let

$$
w(v)=\max \{u:\{u, v\} \in \Sigma\} .
$$

Note that if $v \leq m$, then $\{v, m+1\} \preceq\{m, m+1\} \in \Sigma$, so $w(v) \geq m$. On the other hand, if $v>m$, then $w(v)<v$.

Lemma 10.1. The critical pairs of $\Delta=\operatorname{del}_{1} \Sigma$ are as follows.

For each $v_{1} \in[2, m]$, there is a "type I" critical pair $\left(\left\{v_{1}, v_{2}\right\},\left\{v_{1}, v_{2}+1\right\}\right)$, where $v_{2}=w\left(v_{1}\right)$.

For each $v_{2} \in[m+2, w(2)]$, there is a "type II" critical pair $\left(\left\{v_{1}, v_{2}\right\},\left\{v_{1}+1, v_{2}\right\}\right)$, where $v_{1}=w\left(v_{2}\right)$.

Furthermore, every critical pair is of one of these forms.

Proof. It is immediate from the definition of $w(v)$ that each such pair is critical. Suppose now that $(A, B)$ is a critical pair of $\Delta$, with $A=\left\{v_{1}<v_{2}\right\}$. Then either $B=\left\{v_{1}, v_{2}+1\right\}$ or $B=\left\{v_{1}+1, v_{2}\right\}$.

\footnotetext{
${ }^{5}$ Note the distinction between the variable $X_{i, j}$, which corresponds to vertex $j$ as the $i$ th smallest vertex in a face, and the quadratic monomial $X_{\{i, j\}}$, which corresponds to the edge $\{i, j\}$, and which equals $X_{1, i} X_{2, j}$ if $i \leq j$ but equals $X_{1, j} X_{2, i}$ if $j \leq i$.
} 
If $B=\left\{v_{1}, v_{2}+1\right\}$, then we have already observed that $A$ has at least one endpoint in $[2, m]$. In particular, $v_{1} \leq m$, and the pair $(A, B)$ is of type $\mathrm{I}$.

If $B=\left\{v_{1}+1, v_{2}\right\}$, then $A \in \Delta$ and $B \notin \Delta$, so by definition $v_{1}=w\left(v_{2}\right)$. Moreover, $m+2 \leq v_{2}$ (because $v_{1}+1 \leq v_{2}-1$, so $\left\{v_{2}-1, v_{2}\right\} \succeq B \notin \Delta$ ) and $v_{2} \leq w(2)$ (because $\left\{2, v_{2}\right\} \preceq A$, so $\left.\left\{2, v_{2}\right\} \in \Delta\right)$. Hence the pair $(A, B)$ is of type II.

If $(A, B)$ is a critical pair of type I, then $\sigma(A, B)=\left\{v_{1}, v_{2}\right\}$ and $\bar{\sigma}(A, B)=$ $\left(\left\{v_{1}\right\},\left[2, v_{2}\right]\right)$. If $(A, B)$ is a critical pair of type II, then $\sigma(A, B)=\left\{v_{1}\right\}$ and $\bar{\sigma}(A, B)=\left(\emptyset,\left[2, v_{1}\right]\right)$. Therefore, formula (48) yields

$$
\begin{aligned}
\hat{\tau}_{d}(\Sigma) & =\prod_{v=2}^{n} X_{\{1, v\}} \prod_{v_{1}=2}^{m} \frac{\sum_{j=1}^{w\left(v_{1}\right)} X_{\left\{v_{1}, j\right\}}}{X_{\left\{1, v_{1}\right\}}} \prod_{v_{2}=m+2}^{w(2)} \frac{\sum_{j=1}^{w\left(v_{2}\right)} X_{\{j\}}}{X_{\{1\}}} \\
& =\prod_{v=2}^{n} X_{\{1, v\}} \prod_{v_{1}=2}^{m} \frac{\sum_{j=1}^{w\left(v_{1}\right)} X_{\left\{v_{1}, j\right\}}}{X_{\left\{1, v_{1}\right\}}} \prod_{v_{2}=m+2}^{n} \frac{\sum_{j=1}^{w\left(v_{2}\right)} X_{\{j\}}}{X_{\{1\}}} \\
(50) \quad & X_{\{1, n\}}\left(\prod_{v_{1}=2}^{m} X_{\left\{1, v_{1}\right\}} \frac{\sum_{j=1}^{w\left(v_{1}\right)} X_{\left\{v_{1}, j\right\}}}{X_{\left\{1, v_{1}\right\}}}\right)\left(\prod_{v_{2}=m+2}^{n} X_{\left\{1, v_{2}-1\right\}} \frac{\sum_{j=1}^{w\left(v_{2}\right)} X_{\{j\}}}{X_{\{1\}}}\right) .
\end{aligned}
$$

The second equality follows because $w(v)=1$ whenever $v>w(2)$, and the third equality comes from redistributing most of the first product among the other two. Now, when $v_{2}>m+1$, we have

$$
X_{\left\{1, v_{2}-1\right\}} \frac{\sum_{j=1}^{w\left(v_{2}\right)} X_{\{j\}}}{X_{\{1\}}}=\frac{X_{1,1} X_{2, v_{2}-1}}{X_{1,1}} \sum_{j=1}^{w\left(v_{2}\right)} X_{1, j}=\sum_{j=1}^{w\left(v_{2}\right)} X_{1, j} X_{2, v_{2}-1}=\sum_{j=1}^{w\left(v_{2}\right)} X_{\left\{j, v_{2}-1\right\}}
$$

since $j \leq w\left(v_{2}\right) \leq m<v_{2}-1$. Thus we may rewrite (50) as

$$
\hat{\tau}_{d}(\Sigma)=\left(\prod_{v_{1}=2}^{m} \sum_{j=1}^{w\left(v_{1}\right)} X_{\left\{v_{1}, j\right\}}\right)\left(\prod_{v_{2}=m+2}^{n} \sum_{j=1}^{w\left(v_{2}\right)} X_{\left\{j, v_{2}-1\right\}}\right) X_{\{1, n\}} .
$$

If $v_{1} \leq m$, then $w\left(v_{1}\right)>v_{1}$, so $w\left(v_{1}\right)$ has degree at least $v_{1}$, as does every vertex less than $w\left(v_{1}\right)$. On the other hand, $\left\{v_{1}, w\left(v_{1}\right)+1\right\} \notin \Sigma$, so vertex $w\left(v_{1}\right)+1$ has degree less than $v_{1}$, as does every vertex greater than $w\left(v_{1}\right)+1$. Therefore, $(\operatorname{deg} \Sigma)_{v_{1}}^{\prime}=w\left(v_{1}\right)$.

Similarly, if $v_{2}>m+1$, then $\left(\left\{w\left(v_{2}\right), v_{2}\right\},\left\{w\left(v_{2}\right)+1, v_{2}\right\}\right)$ is a critical pair, so $w\left(v_{2}\right)$ has degree at least $v_{2}-1$, as does every vertex less than $w\left(v_{2}\right)$. On the other hand, vertex $w\left(v_{2}\right)+1$ has degree less than $v_{2}-1$, as does every vertex greater than $w\left(v_{2}\right)+1$. Therefore, $(\operatorname{deg} \Sigma)_{v_{2}-1}^{\prime}=w\left(v_{2}\right)$.

Using these observations to rewrite (51) in terms of the partition $(\operatorname{deg} \Sigma)^{\prime}$ recovers the Martin-Reiner formula (49).

10.3. From threshold graphs to Ferrers graphs. Let $\lambda=\left(\lambda_{1} \geq \cdots \geq \lambda_{\ell}\right)$ be a partition. The corresponding Ferrers graph is the bipartite graph with vertices $x_{1}, \ldots, x_{\lambda_{1}}, y_{1}, \ldots, y_{\ell}$ and edges $\left\{x_{i} y_{j}: i \leq \lambda_{j}\right\}$. That is, the vertices correspond to rows and columns of the Ferrers diagram of $\lambda$, and the edges to squares appearing in the diagram. Ehrenborg and van Willigenburg [14] considered Ferrers graphs and (among other results) described how a certain weighted spanning tree enumerator splits into linear factors. Another proof of their formula can be obtained from the 
foregoing formulas for threshold graphs, as we now explain. The key idea is due to Richard Ehrenborg.

Let $G$ be a connected threshold graph on vertices $[n]$, and let $m$ be the side length of the Durfee square of $G$. Then the vertices $1,2, \ldots, m$ are pairwise adjacent, while $m+1, \ldots, n$ are pairwise nonadjacent. Moreover, if $m+1 \leq i<j \leq n$, then every neighbor of $j$ is a neighbor of $i$. Construct a graph $F$ by deleting all edges $i j$ such that $i, j \leq m$. Then $F$ is a Ferrers graph; furthermore, all Ferrers graphs can be constructed in this way. Thus, if we begin with the weighted enumerator for $G$ and set to zero all indeterminates corresponding to edges between vertices $1,2, \ldots, m$, we recover the weighted enumerator for the corresponding Ferrers graph F. Specifically, Theorem 1.6 yields

$$
\hat{\tau}(G)=X_{1,1} X_{n, 2} \prod_{i=2}^{n-1}\left(\sum_{r=1}^{d_{i}^{T}(G)} X_{\min (i, r), 1} X_{\max (i, r), 2}\right)
$$

(this is also [27, Theorem 4]). Breaking up the product in (52) around the parameter $m$ gives

$$
\begin{array}{r}
\tau(G)=X_{1,1} X_{n, 2}\left(\prod_{i=2}^{m}\left(\sum_{r=1}^{m} X_{\min (i, r), 1} X_{\max (i, r), 2}+\sum_{r=m+1}^{d_{i}^{T}(G)} X_{\min (i, r), 1} X_{\max (i, r), 2}\right)\right) \\
\times\left(\prod_{i=m+1}^{n-1} \sum_{r=1}^{d_{i}^{T}(G)} X_{\min (i, r), 1} X_{\max (i, r), 2}\right) .
\end{array}
$$

This expression is well defined because $d_{i}^{T}(G) \geq m$ whenever $i \leq m$. If $i \leq m$ and $r \leq m$, then $\max (i, r) \leq m$, and these are exactly the terms we wish to set to zero. Therefore,

$$
\tau(F)=X_{1,1} X_{n, 2}\left(\prod_{i=2}^{m} \sum_{r=m+1}^{d_{i}^{T}(G)} X_{i, 1} X_{r, 2}\right)\left(\prod_{i=m+1}^{n-1} \sum_{r=1}^{d_{i}^{T}(G)} X_{\min (i, r), 1} X_{\max (i, r), 2}\right) .
$$

For $i \geq m+1$, we have $d_{i}^{T}(G)<m<i$. Thus $r<i$ for $r \leq d_{i}^{T}(G)$, and (53) yields

$$
\begin{aligned}
& \tau(F)=X_{1,1} X_{n, 2}\left(\prod_{i=2}^{m} X_{i, 1} \prod_{i=2}^{m} \sum_{r=m+1}^{d_{i}^{T}(G)} X_{r, 2}\right)\left(\prod_{i=m+1}^{n-1} X_{i, 2} \prod_{i=m+1}^{n-1} \sum_{r=1}^{d_{i}^{T}(G)} X_{r, 1}\right) \\
&=\left(X_{1,1} X_{2,1} \ldots X_{m, 1}\right)\left(X_{m+1,2} X_{m+2,2} \ldots X_{n, 2}\right) \\
& \times\left(\prod_{i=2}^{m} \sum_{r=m+1}^{d_{i}^{T}(G)} X_{r, 2}\right)\left(\prod_{i=m+1}^{n-1} \sum_{r=1}^{d_{i}^{T}(G)} X_{r, 1}\right) .
\end{aligned}
$$

By construction, the vertex degrees in $G$ and $F$ are related by the formula

$$
\operatorname{deg}_{F}(i)= \begin{cases}\operatorname{deg}_{G}(i)-m & \text { if } 1 \leq i \leq m, \\ \operatorname{deg}_{G}(i) & \text { if } m+1 \leq i \leq n .\end{cases}
$$

To simplify the notation, set $X_{r, 1}=x_{r}$ and $y_{r-m}=X_{r, 2}=y_{r-m}$. (From this perspective, the two partite sets of $F$ correspond to the indeterminates $\left\{x_{1}, \ldots x_{m}\right\}$ 
and $\left\{y_{1}, \ldots y_{n-m}\right\}$. Therefore,

$$
\tau(F)=\left(x_{1} \ldots x_{m}\right)\left(y_{1} \ldots y_{n-m}\right)\left(\prod_{i=2}^{m} \sum_{r=1}^{d_{i}^{T}\left(F_{2}\right)} y_{r}\right)\left(\prod_{i=2}^{n-m} \sum_{r=1}^{d_{i}^{T}\left(F_{1}\right)} x_{r}\right),
$$

which is Theorem 2.1 of [14.

\section{REFERENCES}

[1] Ron Adin, Counting colorful multi-dimensional trees, Combinatorica 12, no. 3 (1992), 247260. MR1195888 (93j:05036)

[2] Eric Babson and Isabella Novik, Face numbers and nongeneric initial ideals, Electron. J. Combin. 11, no. 2 (2004/06), Research Paper \#R25, 23 pp. (electronic) MR2195431 (2007c:05202)

[3] Matthias Beck and Serkan Hoşten, Cyclotomic polytopes and growth series of cyclotomic lattices, Math. Res. Lett. 13, no. 4 (2006), 607-622. MR2250495 (2007h:52018)

[4] L.W. Beineke and R.E. Pippert, Properties and characterizations of $k$-trees, Mathematika 18 (1971), 141-151. MR0288046 (44:5244)

[5] Anders Björner and Gil Kalai, An extended Euler-Poincaré theorem, Acta Math. 161, no. 3-4 (1988), 279-303. MR971798 (89m:52009)

[6] A. Björner, L. Lovász, S.T. Vrećica, and R.T. Živaljević, Chessboard complexes and matching complexes, J. London Math. Soc. (2) 49 (1994), no. 1, 25-39. MR.1253009 (95c:52021)

[7] Ethan Bolker, Simplicial geometry and transportation polytopes, Trans. Amer. Math. Soc. 217 (1976), 121-142. MR0411983 (54:112)

[8] Béla Bollobás, Modern Graph Theory. Graduate Texts in Mathematics, 184. Springer, New York, 1998. MR1633290 (99h:05001)

[9] Arthur Cayley, A theorem on trees, Quart. J. Math. 23 (1889), 376-378.

[10] A.K. Dewdney, Higher-dimensional tree structures, J. Comb. Theory Ser. B 17 (1974), 160169. MR.0369115 (51:5351)

[11] Xun Dong and Michelle L. Wachs, Combinatorial Laplacian of the matching complex, Electron. J. Combin. 9 (2002), no. 1, Research Paper \#R17, 11 pp. (electronic) MR.1912799 (2003g:05131)

[12] Art M. Duval, A relative Laplacian spectral recursion, Electron. J. Combin. 11 (2004/06), no. 2, Research Paper \#R26, 19 pp. (electronic) MR2224939 (2007d:05037)

[13] Art M. Duval and Victor Reiner, Shifted simplicial complexes are Laplacian integral, Trans. Amer. Math. Soc. 354, no. 11 (2002), 4313-4344. MR.1926878(2003j:15017)

[14] Richard Ehrenborg and Stephanie van Willigenburg, Enumerative properties of Ferrers graphs, Discrete Comput. Geom. 32 (2004), no. 4, 481-492. MR2096744 (2005j:05076)

[15] Sara Faridi, The facet ideal of a simplicial complex, Manuscripta Math. 109, no. 2 (2002), 159-174. MR1935027 (2003k:13027)

[16] Miroslav Fiedler and Jiř́ Sedláček, Uber Wurzelbasen von gerichteten Graphen, Časopis Pěst. Mat. 83 (1958), 214-225. MR0097071(20:3551)

[17] Joel Friedman and Phil Hanlon, On the Betti numbers of chessboard complexes, J. Algebraic Combin. 8 (1998), 193-203. MR.1648484 (2000c:05155)

[18] Allen Hatcher, Algebraic Topology. Cambridge University Press, Cambridge, 2002. Also available online at http://www.math.cornell.edu/ hatcher/AT/ATpage.html. MR1867354 (2002k:55001)

[19] Jürgen Herzog and Enzo Maria Li Marzi, Bounds for the Betti numbers of shellable simplicial complexes and polytopes, Commutative algebra and algebraic geometry (Ferrara), 157-167, Lecture Notes in Pure and Appl. Math., 206, Dekker, New York, 1999. MR1702104 (2001b:13017)

[20] Gil Kalai, Enumeration of $Q$-acyclic simplicial complexes, Israel J. Math. 45, no. 4 (1983), 337-351. MR720308 (85a:55006)

[21] Gil Kalai, Algebraic shifting, Computational commutative algebra and combinatorics (Osaka, 1999), 121-163, Adv. Stud. Pure Math., 33, Math. Soc. Japan, Tokyo, 2002. MR1890098 (2003e:52024)

[22] G. Kirchhoff, Über die Auflösung der Gleichungen, auf welche man bei der Untersuchung der linearen Verteilung galvanischer Ströme geführt wird, Ann. Phys. Chem. 72 (1847), 497-508. 
[23] Caroline Klivans, Obstructions to shiftedness, Discrete Comput. Geom. 33 (2005), 535-545. MR2121994 (2005k:06008)

[24] W. Kook, V. Reiner, and D. Stanton, Combinatorial Laplacians of matroid complexes, J. Amer. Math. Soc. 13 (2002), 129-148. MR1697094(2001e:05028)

[25] N.V.R. Mahadev and U.N. Peled, Threshold graphs and related topics. Annals of Discrete Mathematics, 56. North-Holland Publishing Co., Amsterdam, 1995. MR1417258(97h:05001)

[26] Jeremy L. Martin and Victor Reiner, Cyclotomic and simplicial matroids, Israel J. Math. 150 (2005), 229-240. MR2255809 (2007g:05040)

[27] Jeremy L. Martin and Victor Reiner, Factorization of some weighted spanning tree enumerators, J. Combin. Theory Ser. A 104, no. 2 (2003), 287-300. MR2019276 (2004i:05070)

[28] Gregor Masbaum and Arkady Vaintrob, A new matrix-tree theorem, Int. Math. Res. Not. 2002, no. 27, 1397-1426. MR.1908476 (2003a:05107)

[29] J.W. Moon, Counting Labeled Trees. Canadian Mathematical Monographs, No. 1, Canadian Mathematical Congress, Montreal, 1970. MR0274333 (43:98)

[30] Gerald Reisner, Cohen-Macaulay quotients of polynomial rings, Adv. Math. 21 (1976), no. 1, 30-49. MR.0407036 (53:10819)

[31] Jeffrey B. Remmel and S. Gill Williamson, Spanning trees and function classes, Electron. J. Combin. 9, no. 1 (2002), Research Paper \#R34, 24 pp. (electronic) MR 1928786|(2003g:05067)

[32] Richard P. Stanley, Combinatorics and Commutative Algebra, 2nd ed., Birkhäuser, Boston, 1996. MR1453579 (98h:05001)

[33] Richard P. Stanley, Enumerative Combinatorics, volume I. Cambridge Studies in Advanced Mathematics, 49. Cambridge University Press, 1997. MR.1442260 (98a:05001)

[34] Richard P. Stanley, Enumerative Combinatorics, volume II. Cambridge Studies in Advanced Mathematics, 62. Cambridge University Press, 1999. MR.1676282 (2000k:05026)

[35] Vladimir Turaev, Introduction to combinatorial torsions, Lectures in Mathematics ETH Zürich, Birkhäuser Verlag, Basel, 2001. MR.1809561 (2001m:57042)

Department of Mathematical Sciences, University of Texas at El Paso, El Paso, Texas 79968-0514

Departments of Mathematics and Computer Science, The University of Chicago, Chicago, Illinois 60637

Department of Mathematics, University of Kansas, Lawrence, Kansas 66047 